\title{
Nonlinear Dimming and Correlated Color Temperture Control of Bi-Color White LED Systems
}

\author{
Huan-Ting Chen, Member, IEEE, Siew-Chong Tan, Senior Member, IEEE, S. Y. (Ron) Hui, Fellow, \\ IEEE
}

\begin{abstract}
This paper proposes a nonlinear approach of controlling the luminous intensity and correlated color temperature (CCT) of white light-emitting diode (LED) systems with dual color temperatures. This LED system is made up of a warm color LED source $(2700 \mathrm{~K})$ and a cool color LED source $(5000 \mathrm{~K})$. The luminous intensity of each of these LED sources is individually controlled by pulsewidth modulation. The overall intensity of the LED system is due to the combined emitted flux of both LED sources. Its overall CCT is the mixed average CCT of both LED sources. This proposed method is based on the nonlinear empirical luminous and CCT models of the LEDs, which take into consideration the thermal effect of LEDs on its luminance and CCT properties. With reasonable approximation, the theoretical models are simplified into practical solutions, which are translatable into real-life applications. It is experimentally validated that the proposed approach is considerably more accurate than existing linear approaches that do not consider color variations of LED sources. The idea is applicable to LED systems with multiple color temperatures and is not limited to white LEDs.
\end{abstract}

Index Terms - Lighting system, PWM driving, correlated color control (CCT), light-emitting diodes (LED), white LED

\section{INTRODUCTION}

$\mathrm{T}$ he high-power light-emitting diode (LED) is considered an attractive candidate for replacing incandescent and fluorescent lightings for general energy-efficient illumination purpose. There are, however, various aspects that require attention when it comes to applying LEDs for general lighting. One such aspect is related to the LED's junction temperature, which critically affects its operating lifetime,

Manuscript received Jul 29, 2014. This work is supported by the Hong Kong Research Grant Council under Theme-based Research Project: T22-715-12N

H. T. Chen is with the Department of Electrical \& Electronic Engineering, The University of Hong Kong (email: htchen23@ gmail.com).

S. C. Tan is with the Departments of Electrical \& Electronic Engineering, The University of Hong Kong (email: sctan@eee.hku.hk)

S.Y. R. Hui is with the Departments of Electrical \& Electronic Engineering, The University of Hong Kong (email: ronhui@eee.hku.hk) and Imperial College London (e-mail: r.hui@imperial.ac.uk). efficiency, and color properties, and must be properly considered when it comes to designing good-quality light sources [1], [2]. On the other hand, there is also primary concerns of having compatibly efficient and reliable LED ballasts. For this reason, most research works related to LED ballasts so far, have been focused on achieving high energy efficiency [3]-[8], good dimming properties [1], [2], [9]-[14], and high reliability through the avoidance of using electrolytic capacitors [15]-[21]. Recently, it has been pointed out that LEDs with flickering light might be detrimental to human health [22], [23]. Subsequently, a series of work related to achieving good ballast reliability without introducing visible and invisible low-frequency flickering have been reported [24]-[28].

Color perception of the LED light is also an important subject for research in LED systems. In terms of indoor applications, it is important to take into account the correlated color temperature (CCT) of the illumination. Warm-white light sources (i.e., lower CCT) are preferred in public areas to promote relaxation and cool-white light sources (i.e., higher CCT) are used to enhance concentration in offices [29], [30]. For more sophisticated applications as in museums, hotels, boutique shops, etc., a high level of CCT consistency between different lamps is an expectation. Unfortunately, CCT control and dimming is a difficult task in LED technology since binning, age, driving techniques, and temperature drift affect the CCT of the actual emitted light [2], [31]. Therefore, when designing LED ballasts for such applications, factors like the color property and its dimming control should be considered in addition to the basic requirements of achieving high efficiency and reliability. A resulting objective as such would be to achieve LED lamps that can maintain a constant CCT during brightness control and conversely that can maintain constant brightness during CCT adjustment.

Dimmable lamps that have adjustable CCT of wide ranges are currently in good demand [32]. Lamps with such a feature typically allows the continuous change of the CCT from a low value (warm white) to a high value (cool white). In particular, variable CCT LED lamps based on bi-color LED sources are a popular configuration [33], [34], and has been adopted in various products by the lighting industry. In this configuration, the lamp must comprise light sources with at least two distinct CCT values. In the case of LED lamps, an array of LEDs with low CCT (e.g. $2700 \mathrm{~K}$ ) and an array of LEDs with high CCT (e.g. $5000 \mathrm{~K}$ ) may be adopted in the product. If light of $2700 \mathrm{~K}$ is required, only LEDs with CCT of $2700 \mathrm{~K}$ are turned on. If light of $5000 \mathrm{~K}$ is required, only LEDs with CCT of $5000 \mathrm{~K}$ are 
turned on. Subsequently, for light of CCT between $2700 \mathrm{~K}$ and $5000 \mathrm{~K}$, both arrays of LEDs are turned on and driven such that the overall combined light emitted from the lamp is of the required CCT value and brightness.

To perform smooth and continuous brightness control of the lamp, the luminous flux emitted by the individual LED array has to be adjusted. This adjustment can be achieved either by changing the amplitude level or the duty-cycle pulse, or by concurrently changing both the amplitude level and the duty-cycle pulse of the currents flowing through the LEDs [33], [34]. However, the CCT of an LED is dependent on both its junction temperature as well as the amplitude of its drive current [35], [36]. With the two LED sources within the lamp sharing the same heatsink and driven together, but individually controlled, there will be thermal influence of one LED source on the CCT property of the other. In other words, there is mutual thermal interdependence on the CCT of each LED source and such an effect should be carefully treated. For example, if the current of the warm-white LED source is reduced, its junction temperature will be reduced, thereby the heatsink will be cooler. This may indirectly reduce the junction temperature of its companion cool LED source and thereby changing the CCT of the cool-white source. The effect is vice versa for the warm LED source. Hence, the overall CCT control is affected.

Such factors should not be ignored in the design of the bi-color variable CCT white LED lamps. Otherwise, there will always be an undesired change of the CCT in the process of adjusting brightness regardless of the control approach adopted. It must be mentioned that $\mathrm{a} \pm 200 \mathrm{~K}$ deviation within the desired CCT value is often cited as an acceptable error in electric lamps and is considered non eye-perceivable. Nevertheless, it is found that existing approaches do not take into consideration the abovementioned factors and possess huge perceivable CCT deviation within their CCT control [33], [34], as will be illustrated in the experimental works in the later section.

In view of this, the work in this paper is primarily targeted at achieving accurate dimming and CCT control of bi-color variable CCT LED lamps, through the use of a nonlinear empirical LED model that accounts the thermal interdependency effect of the two color sources and the actual imperfections of LEDs. The system described is based on a white LED lamp configured with LEDs of two different color temperatures, namely the $2700 \mathrm{~K}$ and $5000 \mathrm{~K}$ types of LEDs. Nevertheless, the idea is also applicable to LED systems with multiple color temperatures and is not limited to white LEDs. However, it must be emphasized that this proposed method [37] does not take into consideration the aging effect of LEDs, which can be mitigated through feedback sensor, and is beyond the scope of this discussion. Section II gives an overview of the existing issue of wide CCT variation of common phosphor-converted white LED during brightness control, and discusses why the linear approaches of controlling bi-color white LED lamps are still incapable of achieving satisfactory CCT control performance. Section III gives a description of the proposed nonlinear control approach. Section IV provides the experimental results and discussion. Finally, Section V gives the conclusion of the paper..

\section{ISSUES OF PHOSPHOR-CONVERTED WHITE LED SYSTEMS}

\section{A. Wide CCT Variation of Phosphor-Converted White LED Systems}

Currently, the two most popular techniques of driving LEDs are DC drive and pulsewidth modulation (PWM) drive. In DC drive (also known as amplitude mode drive), the current driving the LED is a DC constant current, of which the luminous intensity of the LED is changed by varying the amplitude of current. In PWM drive, the current driving the LED is a current pulsating between constant amplitude and zero current. Brightness control of the LED is typically changed by varying the duty-cycle ratio of the pulsating current [22]. Due to the nonlinear I-V characteristic of the LED, PWM drive results in better linear control of the light intensity as compared to DC drive. However, driving LEDs using PWM techniques introduce additional efficacy loss that is otherwise not present with DC driving methods [9], [22]. This loss is converted into additional heat which further aggravates the undesired chromaticity-shifting trait of LEDs [2], [37]. The effect of dimming and heat on chromaticity shift of LEDs has been reported in [2], [37], [38]. In particular, with DC drive, the peak wavelength of LEDs shows a blue shift with increased light intensity (i.e., increased current level), which is attributed to the band filling and quantum confined stark effects [39]. With PWM drive, LEDs exhibit a red shift with increased light intensity (i.e., increased duty cycle), which is dominantly cause by the increase in junction heat [40]. Despite the various reports on the dimming effects of DC and PWM drives on the chromaticity property of LEDs in terms of peak-wavelength and CIE coordinates, as far as we know, no literature has been published comparing the effect of dimming on the CCT shift of phosphor-converted (PC) white LED for DC and PWM drives, under the same luminous flux output and with consideration to the use of different heatsinks sizes. Additionally, in order to further clarify the misconception that color consistency during dimming is better with PWM drive than DC drive, experimental results of a PC white LED in terms of the CCT versus the luminous flux for PWM drive and DC drive for different heatsinks, are given in Fig. 1.

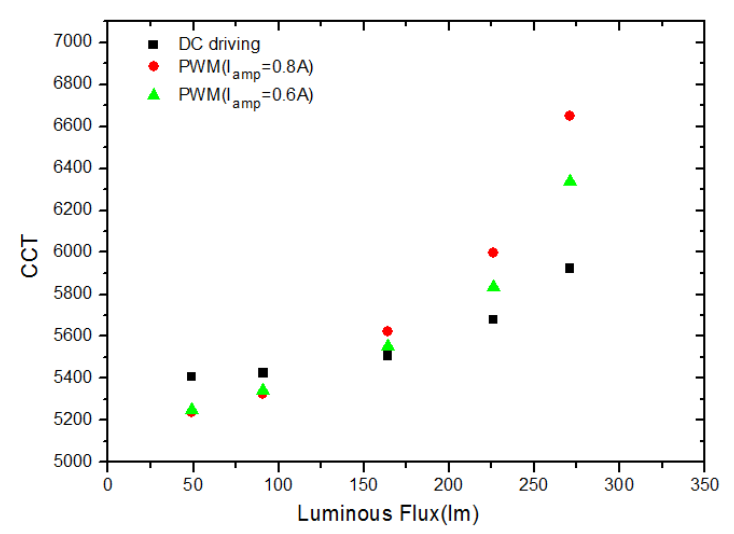

Fig. 1(a) $9.7 \mathrm{~K} / \mathrm{W}$ 


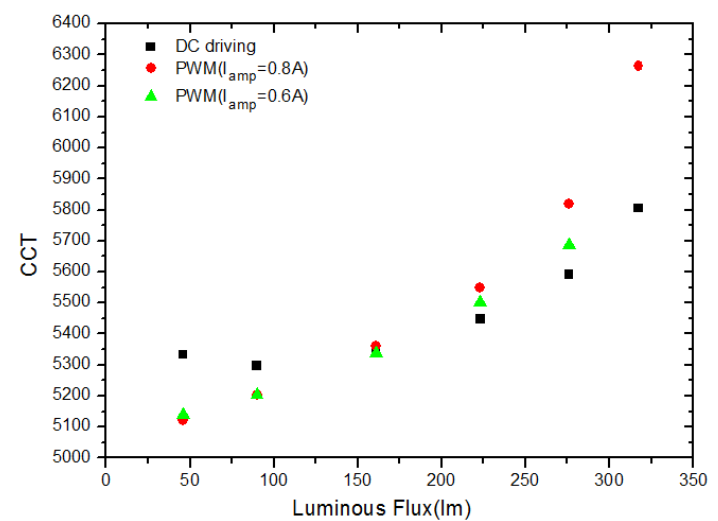

Fig. 1(b) $6.3 \mathrm{~K} / \mathrm{W}$

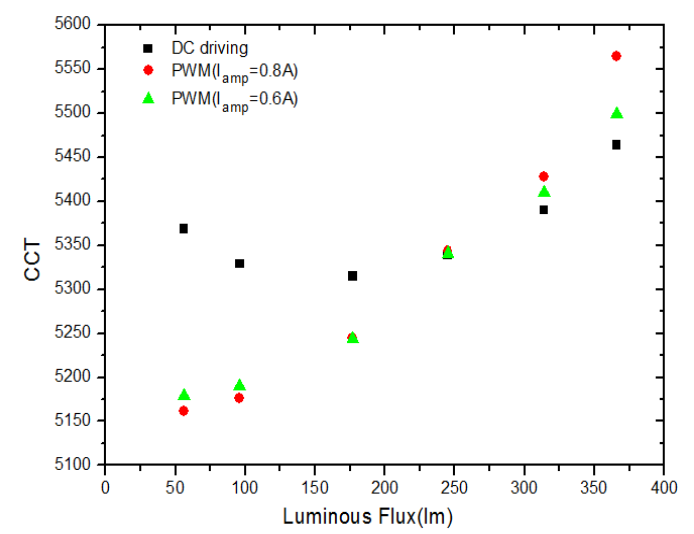

Fig. $1(\mathrm{c}) 1.7 \mathrm{~K} / \mathrm{W}$

Fig. 1. Experimental results of CCT variation of PC white LEDs versus its luminous flux under DC drive with variable current amplitude and PWM driving methods with amplitude currents of $0.6 \mathrm{~A}$ and $0.8 \mathrm{~A}$, under thermal resistance of (a) 9.7 $\mathrm{K} / \mathrm{W}$, (b) $6.3 \mathrm{~K} / \mathrm{W}$, and (c) $1.7 \mathrm{~K} / \mathrm{W}$.

In the experiments, both the DC and PWM drives are applied to the same LED (a Sharp 4.4 W, GW5BNC15L02) for fairness of comparison. Three sets of experiments, each with the LED mounted on a different heatsink, with thermal resistance of respectively $9.7 \mathrm{~K} / \mathrm{W}$ (see Fig. 1(a)), $6.3 \mathrm{~K} / \mathrm{W}$ (see Fig. 1(b)), and $1.7 \mathrm{~K} / \mathrm{W}$ (see Fig. 1(c)) are performed. The ambient temperature is kept constant at $25^{\circ} \mathrm{C}$ and the heatsink operates under free convection with no active temperature control ${ }^{1}$. For each set of experiment, the DC drive and the PWM drive using amplitude currents of $0.6 \mathrm{~A}$ and $0.8 \mathrm{~A}$ are employed. The dimmable luminous-flux range of the LED with heatsink's thermal resistance of $9.7 \mathrm{~K} / \mathrm{W}, 6.3 \mathrm{~K} / \mathrm{W}$, and $1.7 \mathrm{~K} / \mathrm{W}$ are respectively from $50 \mathrm{~lm}$ to $270 \mathrm{~lm}$, from $50 \mathrm{~lm}$ to $320 \mathrm{~lm}$, and from $50 \mathrm{~lm}$ to $366 \mathrm{~lm}$.

Table. 1. Experimental results of CCT variation of PC white LEDs versus its luminous flux under DC drive with variable current amplitude and PWM driving methods

\footnotetext{
${ }^{1}$ All experiments hereon are conducted at ambient temperature of $25{ }^{\circ} \mathrm{C}$ under free convection without active temperature control.
}

\begin{tabular}{|c|c|c|c|c|}
\hline \multirow{4}{*}{$\begin{array}{c}\text { Current } \\
\text { amplitude( } \\
\text { A) }\end{array}$} & $\begin{array}{c}\text { Heatsink's } \\
\text { thermal } \\
\text { resistance(K/ } \\
\mathrm{W})\end{array}$ & $\begin{array}{c}\text { Dimmable } \\
\text { luminous-fl } \\
\text { ux range } \\
\text { (lm) }\end{array}$ & $\begin{array}{c}\text { CCT } \\
\text { variatio } \\
\text { n (K) }\end{array}$ \\
\hline \multirow{4}{*}{$\begin{array}{c}\text { PW } \\
\text { drive }\end{array}$} & 0.6 & 9.7 & $50-270$ & 1088 \\
\cline { 2 - 5 } & 0.8 & 9.7 & $50-270$ & 1413 \\
\cline { 2 - 5 } & 0.6 & 6.3 & $50-320$ & 548 \\
\cline { 2 - 5 } & 0.8 & 6.3 & $50-320$ & 697 \\
\hline \multirow{2}{*}{$\begin{array}{c}\text { DC } \\
\text { drive }\end{array}$} & 0.6 & 1.7 & $50-366$ & 325 \\
\cline { 2 - 5 } & - & 1.7 & $50-366$ & 403 \\
\cline { 2 - 5 } & - & 9.7 & $50-270$ & 514 \\
\hline \multirow{2}{*}{} & - & 6.3 & $50-320$ & 245 \\
\hline
\end{tabular}

As shown in Fig. 1(a) and Table. 1, the experimental results are $149 \mathrm{~K}$ (DC drive), $403 \mathrm{~K}$ (PWM, $0.8 \mathrm{~A}$ amplitude), and $325 \mathrm{~K}$ (PWM, 0.6 A amplitude). Experimental results showed that the CCT variation under PWM drive is much larger than that under DC drive. Also, in all cases of PWM drive, the CCT variation is eye-perceivable when the LED is fully dimmed since the variations are all greater than $200 \mathrm{~K}$. For DC drive, CCT variation is non-perceivable when the heatsink is very large with a thermal resistance of $1.7 \mathrm{~K} / \mathrm{W}$. Otherwise, CCT variation of LEDs under DC drive is still perceivable during full range dimming when mounted on more regular sized heatsinks. Note that the analyses above are true for cool-white PC LED, of which the CCT is high and widely varied. In the case of warm-white PC LED of which the CCT is low, the CCT variation is typically small and non-perceivable during dimming. Nevertheless, in applications requiring cool color of a constant CCT during dimming, the single-colored cool-white PC LED array, regardless of the driving method, will be inapplicable. In such circumstances, the bi-color white LED system with automatic CCT control may be adopted. In addition, a bi-color white LED system also allows wide CCT adjustment and light intensity control, which is unachievable with the single-colored type of PC LED systems.

\section{B. Existing Linear Control Approaches for Bi-Color Variable CCT White LED Systems}

In general, the bi-color variable CCT white LED systems comprise two LED sources, with each emitting light of a different CCT [33], [34]. The system includes a controller which allows the adjustment of the overall light intensity and CCT of the lamp. This is dictated through the individual adjustment of the light intensity of each LED array. Currently, approaches adopted in performing the CCT mixing in such lamps with bi-color LED sources are generally based on simple linear CCT averaging of the two LED sources. For example, in the method proposed in [33], lights of the two LED sources are mixed to give a desired CCT by controlling the proportion of the emitted light of each respective source. In the method proposed in [34], the two LED sources are placed in anti-parallel manner such the anode of one LED source is connected to the cathode of the other LED source and vice versa. Current flowing in one direction turns on the first LED 
source and current flowing in the opposite direction turns on the second LED source. A controller manages the control of the CCT by adjusting the duty ratio of an alternating current flowing through the two LEDs to control the CCT and/or the brightness of the lighting apparatus.

In both these approaches, the control of the CCT and flux of the LED lamp is based on the mathematical expression

$$
\left\{\begin{array}{l}
C C T_{M}=C C T_{W} D+C C T_{C}(1-D)=C C T_{W} \frac{\phi_{W}}{\phi_{W}+\phi_{C}}+C C T_{C} \frac{\phi_{C}}{\phi_{W}+\phi_{C}} \\
\phi_{M}=\phi_{W}+\phi_{C}
\end{array}\right.
$$

where $C C T_{\mathrm{M}}$ and $\phi_{M}$ are respectively the CCT value and overall flux of the total light mixture from the lamp, $\phi_{w}$ and $C C T_{\mathrm{W}}$ are respectively the averaged luminous flux and CCT value of the warm-white LED source, $\phi_{C}$ and $C C T_{\mathrm{C}}$ are respectively the luminous flux and CCT value of the cool-white LED source, and $D$ is the ratio of the averaged emitted warm color light flux $\phi_{w}$ over the total light flux of the lamp (i.e., $\left.\phi_{W}+\phi_{C}\right)$. Here, $C C T_{W}$ and $C C T_{C}$ are assumed to be constant, which is untrue in practice. Due to this assumption, $C C T_{\mathrm{M}}$ is a simple linear combination of $C C T_{W}$ and $C C T_{C}$ with regards to the change of $D$, and for this reason, approaches of this nature are all fundamentally linear approaches.

The main problem with linear approaches are that they are rather simplistic considering that non-ideal characteristics such as the effect of temperature change on CCT and luminance, and the thermal interdependency effect between the two LED sources, are all neglected. Without proper consideration to the actual dynamics of luminous flux, CCT, current, temperature, and duty cycle change, the achievable CCT control will be highly inaccurate. Such errors are significant especially if wide-range dimming and CCT control are required since the temperature variation in such operations are large. Figs. 2 and 3 give the experimental proofs of the stated problem.

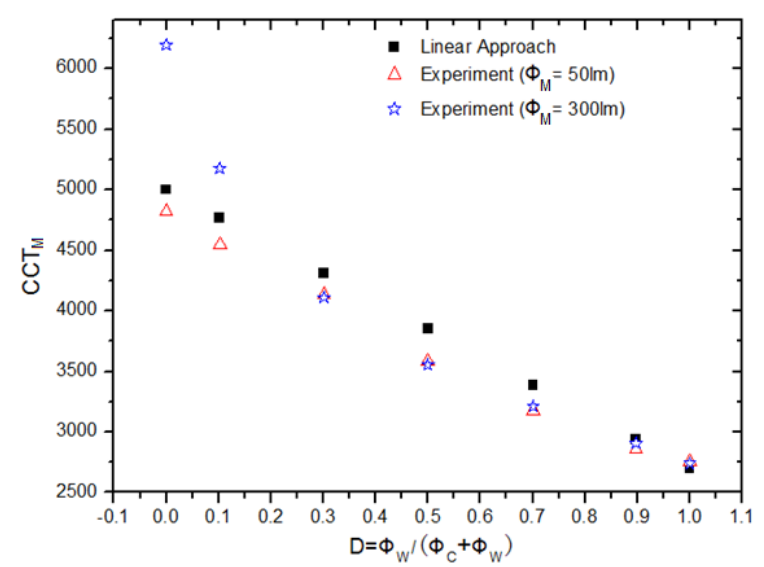

Fig. 2. Experimentally measured and calculated values of $C C T_{M}$ of bi-color white LED system at different $D$ for $\phi_{M}=50$ $\mathrm{lm}$ and $\phi_{M}=300 \mathrm{~lm}$.

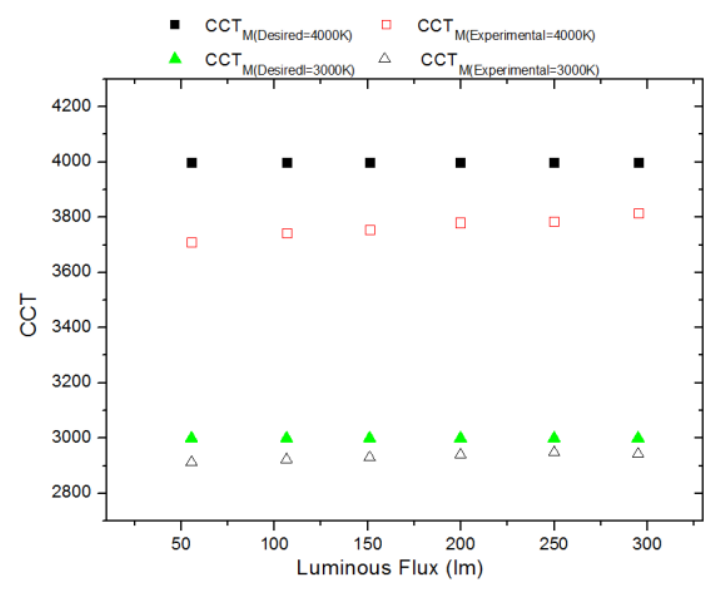

Fig. 3. Experimentally measured values of $C C T_{M}$ for different luminous flux $\phi_{M}$ for two preset CCT references of $C C T_{M(\text { Desired })}=3000 \mathrm{~K}$ and $4000 \mathrm{~K}$ under the linear approach.

In the experiments, a cool-white PC LED (Sharp GW5BNC15L02) and a warm-white PC LED (Sharp GW5BTF27K00) making up the bi-color white LED lamp are mounted on a heatsink with thermal resistance of $6.3 \mathrm{~K} / \mathrm{W}$ and driven by DC currents. According to the datasheets provided, the typical CCT of GW5BNC15L02 is $5000 \mathrm{~K}$ and that of GW5BTF27K00 is $2700 \mathrm{~K}$. For linear approaches, these will be the values adopted for the $\mathrm{CCT}$ of the respective sources, that is, $C C T_{\mathrm{C}}=5000 \mathrm{~K}$ and $C C T_{\mathrm{W}}=2700 \mathrm{~K}$.

Fig. 2 shows the results of the experiment conducted to measure the CCT value of the lamp, i.e., $C C T_{M}$, for different $D$ and at two different luminance levels of $\phi_{M}=50 \mathrm{~lm}$ and $\phi_{M}=$ $300 \mathrm{~lm}$. The results plotted as "Linear Approach" is that calculated using equation (1). Predictably, with linear approaches, the value of $C C T_{M}$ reduces linearly with an increasing $D$, irrespective of the luminance level of the lamp. However, the measured CCT value of the lamp shows otherwise. Not only does $C C T_{M}$ differ at different luminance levels, they do not change linearly with $D$. Moreover, at high level of CCT and high luminance level, the CCT deviation is higher. A maximum CCT error of $1400 \mathrm{~K}$ is found at $D=0$ and $\phi_{M}=300 \mathrm{~lm}$.

Table. 2. Experimental and desired CCT for different luminous flux

\begin{tabular}{|c|c|c|c|c|}
\hline $\begin{array}{l}\text { Bi-colo } \\
\text { r white } \\
\text { LED } \\
\text { lamp }\end{array}$ & $\begin{array}{l}C C T_{M(\text { Desired }} \\
(\mathrm{K})\end{array}$ & $D$ & $\begin{array}{l}\text { Luminou } \\
\text { s Flux } \\
(\mathrm{lm})\end{array}$ & $\begin{array}{l}C C T_{M(\text { Experimental }} \\
(\mathrm{K})\end{array}$ \\
\hline \multirow{2}{*}{$\begin{array}{l}C C T_{\mathrm{C}}= \\
5000 \mathrm{~K}, \\
C C T_{\mathrm{W}}= \\
2700 \mathrm{~K}\end{array}$} & 3000 & $\begin{array}{l}0.86 \\
9\end{array}$ & $\begin{array}{l}50 \text { and } \\
300\end{array}$ & 2913 and 2946 \\
\hline & 4000 & $\begin{array}{l}0.43 \\
4\end{array}$ & $\begin{array}{l}50 \text { and } \\
300\end{array}$ & 3710 and 3814 \\
\hline
\end{tabular}

Fig. 3 and Table 2 show the results of how the CCT value of the lamp changes with the change in luminance level.. By using these values in the adjustment of the luminance level of the individual LED sources and then measuring the value of $C C T_{M}$, it is evident from the data that there is some deviation between the desired CCT and the actual experimental CCT of 
the lamp. In the following section, a nonlinear dimming and CCT approach is proposed to reduce these errors in the control of the lamp.

\section{PROPOSED NONLINEAR DIMMING AND CCT CONTROL APPROACH}

The essence of the proposed nonlinear dimming and CCT control approach is that by taking into consideration $C C T_{\mathrm{W}}$, $C C T_{\mathrm{C}}, \phi_{C}$, and $\phi_{W}$ are all nonlinear functions of current level, duty ratio, junction temperature, heatsink's thermal resistance, and the thermal interdependency effect of the two LED sources, a more precise dimming and CCT control performance is achievable.

\section{A. Nonlinear CCT function of Bi-Color Variable CCT White LED Systems}

According to colorimetry, the relationship between the tristimulus values $(X, Y, Z)$ and chromaticity $(x, y, z)$ of a light source can be written as

$$
\left\{\begin{array}{l}
x=\frac{X}{X+Y+Z} \\
y=\frac{Y}{X+Y+Z} \\
z=\frac{Z}{X+Y+Z}
\end{array}\right.
$$

Equation (2) can be rewritten as

$$
\frac{X}{x}=\frac{Y}{y}=\frac{Z}{z}=X+Y+Z
$$

or

$$
\left\{\begin{array}{l}
X=\frac{x}{y} Y \\
Z=\frac{z}{y} Y=\frac{1-x-y}{y} Y
\end{array}\right.
$$

If the tristimulus values for the warm-white LED source is $\left(X_{W}, Y_{W}, Z_{W}\right)$ and for the cool-white LED source is $\left(X_{C}, Y_{C}, Z_{C}\right)$, the overall tristimulus values of the light emitted from the bi-color LED lamp, which is the sum of the respective sources, is

$$
\left\{\begin{array}{l}
X_{M}=X_{W}+X_{C} \\
Y_{M}=Y_{W}+Y_{C} \\
Z_{M}=Z_{W}+Z_{C}
\end{array} .\right.
$$

Therefore, the overall chromaticity coordinates of the bi-color LED system are given by

$$
\begin{aligned}
& x_{M}=\frac{X_{M}}{X_{M}+Y_{M}+Z_{M}}=\frac{X_{W}+X_{C}}{X_{W}+X_{C}+Y_{W}+Y_{C}+Z_{W}+Z_{C}} \\
& =\frac{X_{W}+X_{C}}{X_{W}+X_{C}+\frac{y_{w}}{x_{w}} X_{W}+\frac{y_{C}}{x_{C}} X_{C}+\frac{1-x_{w}-y_{w}}{x_{w}} X_{W}+\frac{1-x_{c}-y_{c}}{x_{c}} X_{C}} \\
& =\frac{X_{W}+X_{C}}{\frac{X_{W}}{x_{w}}+\frac{X_{C}}{x_{c}}}
\end{aligned}
$$

$$
\begin{aligned}
& y_{M}=\frac{Y_{M}}{X_{M}+Y_{M}+Z_{M}}=\frac{Y_{W}+Y_{C}}{X_{W}+X_{C}+Y_{W}+Y_{C}+Z_{W}+Z_{C}} \\
& =\frac{Y_{W}+Y_{C}}{\frac{x_{w}}{y_{w}} Y_{W}+\frac{x_{c}}{y_{c}} Y_{C}+Y_{W}+Y_{C}+\frac{1-x_{w}-y_{w}}{y_{w}} Y_{W}+\frac{1-x_{c}-y_{c}}{y_{c}} Y_{C}} \\
& =\frac{\frac{Y_{W}+Y_{C}}{Y_{W}}+\frac{Y_{C}}{y_{c}}}{y_{w}}
\end{aligned}
$$

Since the tristimulus value $Y$ represents luminance, which is a proportional to the luminous flux $\Phi_{v}$, and chromaticity $y$ can be approximated as being proportional to CCT, as shown in Fig 4, therefore, equation (6.2) can be rewritten as

$$
C C T_{M}=\frac{\phi_{W}+\phi_{C}}{\frac{\phi_{W}}{C C T_{W}}+\frac{\phi_{C}}{C C T_{C}}}
$$

Equation (7) represents a nonlinear function of the mixed $C C T_{M}$ linking luminous flux and CCT of individual LED sources together with that of the mixed light, for a general bi-color white LED system. Unlike in linear approaches based on equation (1) of which $C C T_{W}$ and $C C T_{C}$ are assumed constant, here $C C T_{W}$ and $C C T_{C}$ are functions of its operating conditions, i.e., current, junction temperature, and ratio $D$. This is important since in practice, the CCT of an LED source is highly dependent on its junction temperature and current amplitude. 


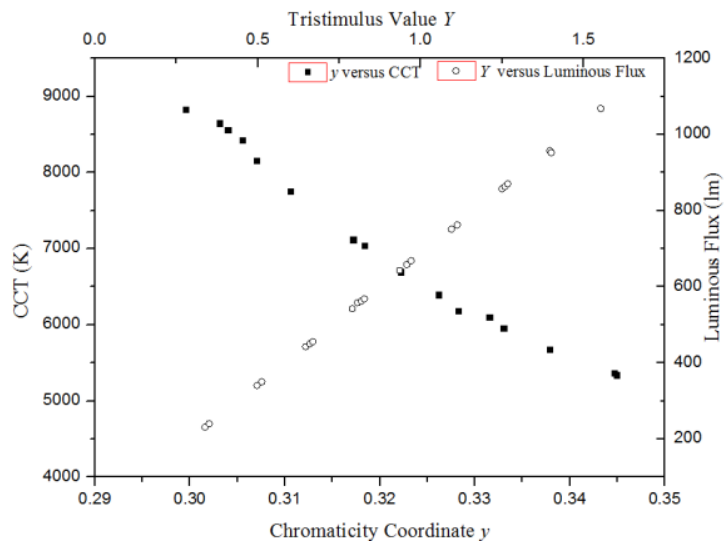

Fig. 4. Measured tristimulus value $Y$ versus luminous flux and measured chromaticity coordinate $y$ versus CCT.

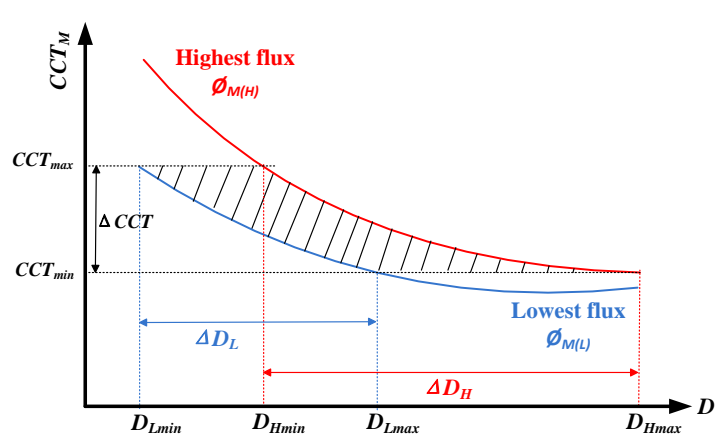

Fig. 5. Typical nonlinear CCT behavior of the bi-color white LED system for different $D$ at high and low luminous flux.

Fig. 5 depicts the typical nonlinear CCT behavior of a bi-color white LED system for different values of $D=$ $\phi_{w} /\left(\phi_{w}+\phi_{C}\right)$ with its highest and lowest total luminous flux, i.e., $\phi_{M(H)}$ and $\phi_{M(L)}$, respectively. As illustrated in the diagram, full range dimming (from $\phi_{M(H)}$ to $\phi_{M(L)}$ ) and CCT control of the lamp is achievable only for the range $\triangle C C T$ (from $C C T_{\min }$ to $C C T_{\max }$ ) through the ratio range of $\Delta D_{L}$ (from $D_{L \min }$ to $D_{\text {Lmax }}$ ) in the case of the lowest flux and $\Delta D_{H}$ (from $D_{H \text { min }}$ to $D_{\text {Hmax }}$ ) in the case of the highest flux. This is represented by the shaded area in the figure. No dimming actions can be performed at CCT below $C C T_{\min }$ and that above $C C T_{\max }$, and that the CCT range is restricted within these values. These boundaries represent the operational dimming and CCT limits of a dimmable bi-color variable CCT white LED lamp.

\section{B. Empirical Luminous Model of the LEDs in the Bi-Color White LED Lamp}

It is known that the junction temperature of an LED affects its luminous efficacy and CCT. Typically, the junction temperature of an LED is affected by its current level, driving technique, heatsink size, and ambient temperature. In the case of the bi-color white LED lamp, the junction temperature of the cool-white LED source will be affected by the operating state of the warm-white LED source since heat of the latter will affect the temperature of the heatsink, which in turns affect the junction temperature of the former, and this is conversely true.
Such a thermal interdependency effect will be accounted in the proposed empirical luminous and CCT models.

(i).Experimental Measurement: The luminous models are built upon results obtained from the LED sources through the following experimental steps. Firstly, with both the cool-white and warm-white LED sources mounted on the same heatsink, the current amplitude of both sources are set at $0.5 \mathrm{~A}$. For both sources, PWM drive is adopted. Then, the warm-white LED is covered using a black rubber which prevents its luminous flux from being emitted into space. What this means is that when the warm-white LED is driven, it only contributes thermal energy into the heatsink. Since the minimum and maximum thermal energy that the warm-white LED can contribute to the LED lamp are generated when it is fully turned off at $D_{W}=0$ and when it is fully turned on at $D_{W}=1$, respectively, the measurement of the luminous characteristic of the cool-white LED with regards to the operating condition of the warm-white LED will be performed for both these boundary conditions. Fig. 6(a) shows the luminous flux versus duty ratio $D_{C}$ of the cool-white LED in both conditions of $D_{W}=0$ and $D_{W}=1$. Clearly, the maximum and minimum luminous flux emission from the cool-white occurs at respectively $D_{W}=0$ and $D_{W}=1$ as heat energy contribution by the warm-white LED is respectively at its lowest and highest. The operating range of the cool-white LED at different operating condition of the warm-white LED (i.e., $0<D_{W}<1$ ) should occur between maximum and minimum curves. By swopping the placement of the black rubber onto the cool-white LED, the same measurement process is repeated on the warm-white LED. Fig. 6(b) shows the luminous flux versus duty ratio $D_{W}$ of the warm-white LED in both conditions of $D_{C}=0$ (cool-white turned off) and $D_{C}=1$ (cool-white fully turned on).

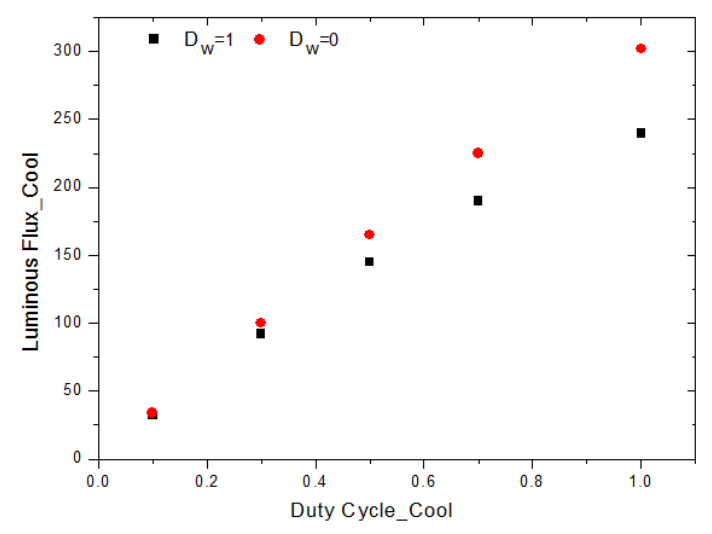

Fig. 6(a)Cool-white LED 


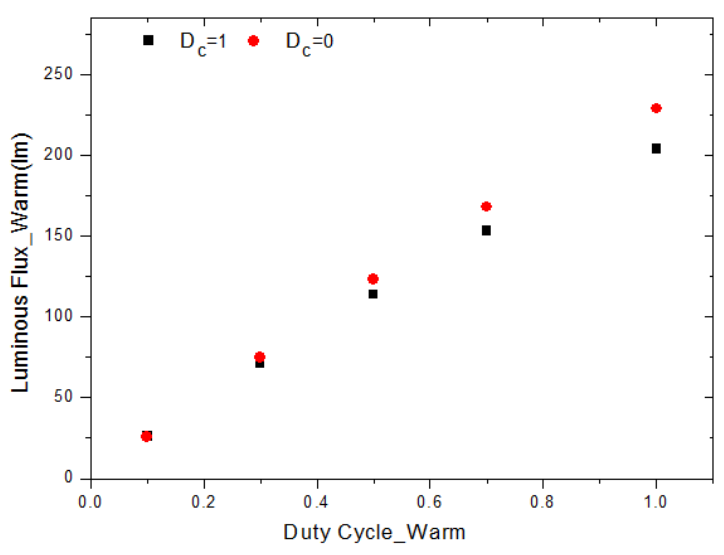

Fig.6. (b) Warm-white LED

Fig. 6. Experimental values of luminous flux of the (a) cool-white LED when the warm-white LED is fully off at $D_{W}=$ 0 and fully on at $D_{W}=1$ and (b) warm-white LED when the cool-white LED is fully off at $D_{C}=0$ and fully on at $D_{C}=1$.

(ii) Exponential Function Curve Fitting: Careful observation of the results shows that the luminous flux characteristics of the cool-white LED with consideration to the thermal contribution from the warm-white LED (as given in Fig. 6(a)) can be modeled using an exponential function of the following form

$$
\phi_{C}=\phi_{C 0}-\alpha_{C} e^{\beta_{C} D_{C}}
$$

where $\phi_{C}$ is luminous flux of cool-white LED, $\phi_{C 0}$ and $\alpha_{C}$ are constant parameters derivable from measurement, and $\beta_{C}$ is a variable related to the duty cycle of warm-white LED, i.e., $D_{W}$. Likewise, the characteristics of the warm-white LED can be expressed as

$$
\phi_{W}=\phi_{W 0}-\alpha_{W} e^{\beta_{W} D_{W}}
$$

Both equations (8a) and (8b) can be rewritten as

$$
\begin{gathered}
\ln \left[\phi_{C 0}-\phi_{C}\right]=\beta_{C} D_{C}+\ln \alpha_{C} \\
\ln \left[\phi_{W 0}-\phi_{W}\right]=\beta_{W} D_{W}+\ln \alpha_{W}
\end{gathered}
$$

Clearly, for any fixed $\beta_{C}$, the luminous flux variation of $\ln \left[\phi_{C 0}-\phi_{C}\right]$ is a linear function of $D_{C} \cdot \beta_{C}$ is a negative parameter that describes the rate of reduction in $\ln \left[\phi_{C 0}-\phi_{C}\right]$ with respect to an increasing $D_{C}$. For this cool-white LED sample, $\phi_{C 0}$ is around $500 \mathrm{~lm}$. The rule of thumb for choosing $\phi_{C 0}$ is to set it at $3 \times \phi_{C}$ when $D_{C}=0.5$. As illustrated in Fig. 7 , the experimental values of $\ln \left[\phi_{C 0}-\phi_{C}\right]$ against the change of $D_{C}$ is basically linear for both cases of $D_{W}=0$ and $D_{W}=1$. Similarly, by setting $\phi_{W 0}=370 \mathrm{~lm}\left(3 \times \phi_{W}\left(D_{W}=0.5\right)\right)$, the experimental values of $\ln \left[\phi_{W 0}-\phi_{W}\right]$ against the change of $D_{W}$ can be calculated for both cases of $D_{C}=0$ and $D_{C}=1$, and are plotted as shown in Fig. 8. The linear relationship of $\ln \left[\phi_{W 0}-\phi_{W}\right]$ against $D_{W}$ is observed.

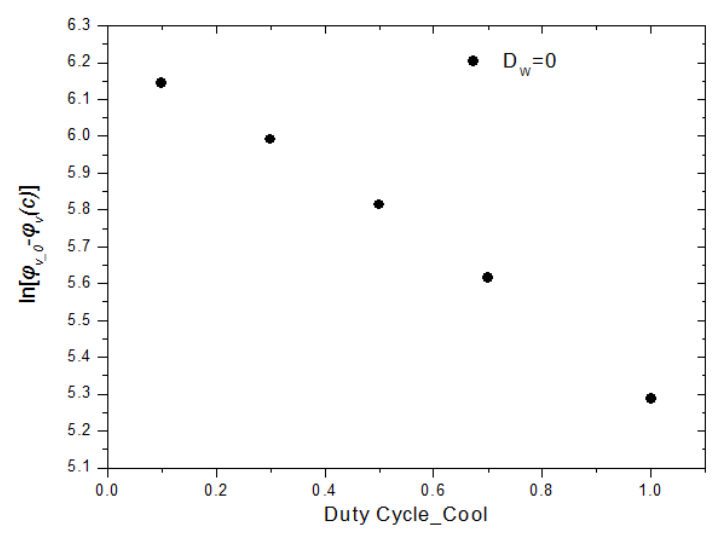

Fig.7. (a) $D_{W}=0$

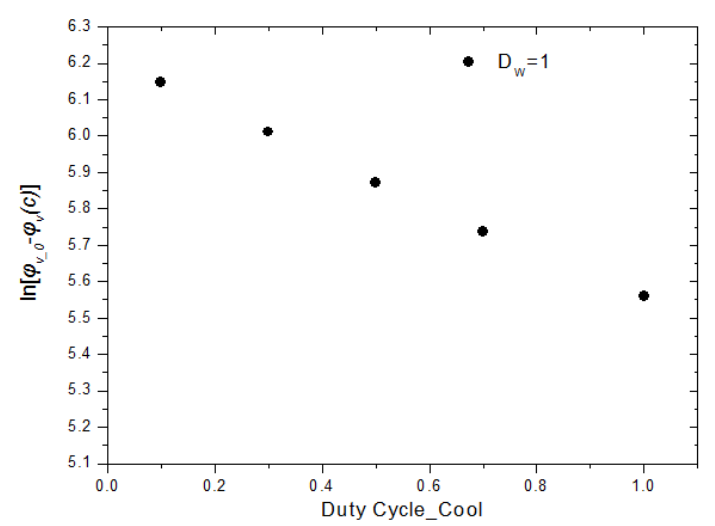

Fig.7. (b) $D_{W}=1$

Fig. 7. Experimental values of $\ln \left[\phi_{C 0}-\phi_{C}\right]$ against duty cycle for the cool-white LED at (a) $D_{W}=0$ and (b) $D_{W}=1$.

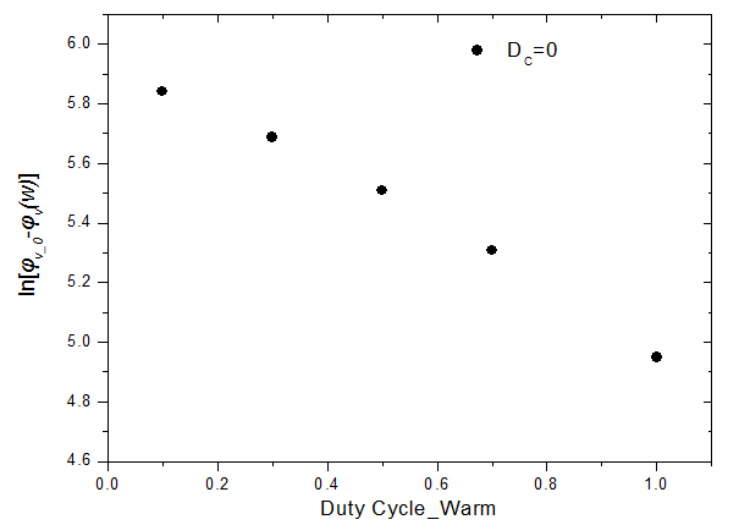

Fig.8. (a) $D_{C}=0$

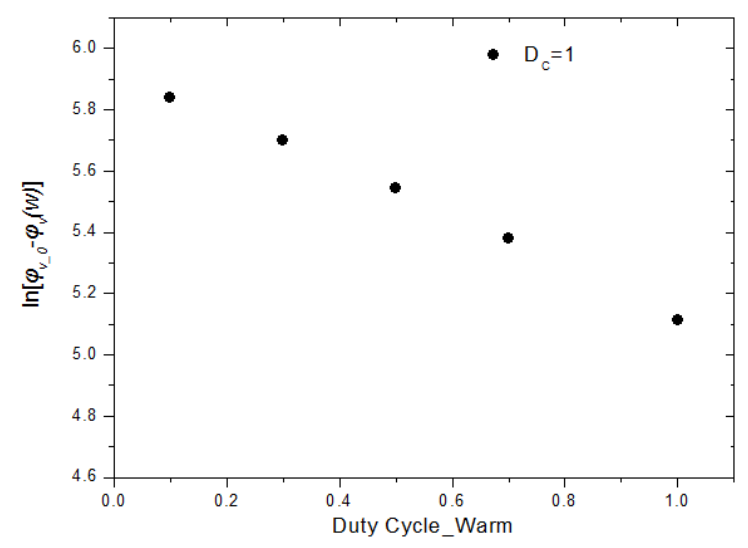


Fig.8. (b) $D_{C}=1$

Fig. 8. Experimental values of $\ln \left[\phi_{W 0}-\phi_{W}\right]$ against duty cycle for the warm-white LED at (a) $D_{C}=0$ and (b) $D_{C}=1$.

The results given in Figs. 7 and 8 can be fitted into straight lines and assume the expressions given in (9a) and (9b), respectively. Both the fitted lines in Figs. 7(a) and 7(b) cross the $y$ axis at the same constant point $\ln \alpha_{C}$ (cool-white LED) and that of Figs. 8(a) and 8(b) at $\ln \alpha_{W}$ (warm-white LED). The values of $\alpha_{C}$ and $\alpha_{W}$ can be derived from these results. The gradient of each of these lines represents the value of $\beta_{x}$, of which in the case of the cool-white LED source, an operating range of $D_{W, \text { min }} \leq D_{W} \leq D_{W, \text { max }}$ for its companion warm-white LED source will generate a range of gradient values of $\beta_{C, \min } \leq \beta_{C} \leq \beta_{C, \max }$. Likewise, for the warm-white LED source, an operating range of $D_{C, \min } \leq D_{C} \leq D_{C, \max }$ for its companion cool-white LED source will generate a range of gradient values of $\beta_{W, \text { min }} \leq \beta_{W} \leq \beta_{W, \text { max }}$. Through the linear association of $D_{W}$ with $\beta_{C}$ and $D_{C}$ with $\beta_{W}$, the luminous flux expression of the cool-white and warm-white LED sources with respect to $D_{C}$ and $D_{W}$ can be respectively expressed as

$\phi_{c}\left(D_{C}, D_{W}\right)=\phi_{C 0}-\alpha_{C} \exp \left\{\left[\frac{\left(\beta_{C, \text { max }}-\beta_{C, \text { min }}\right)}{\left(D_{W, \text { max }}-D_{W, \text { min }}\right)}\left(D_{W}-D_{W, \text { min }}\right)+\beta_{C, \min }\right] D_{C}\right\}$

$\phi_{W}\left(D_{C}, D_{W}\right)=\phi_{W 0}-\alpha_{W} \exp \left\{\left[\frac{\left(\beta_{W, \max }-\beta_{W, \min }\right)}{\left(D_{C, \max }-D_{C, \min }\right)}\left(D_{C}-D_{C, \min }\right)+\beta_{W, \min }\right] D_{W}\right\}$

Equation (10a) gives the luminous flux of the cool-white LED at any $D_{C}$ and $D_{W}$ value, of which $D_{W}$ contributes to the thermal energy affecting the junction temperature of the cool-white LED. Here, the gradient $\beta_{C}$ for any $D_{W}$ is obtained through the linear interpolation of $\beta_{C, \max }$ and $\beta_{C, \min }$. Equation (10b) is the counterpart equation for the warm-white LED.

(iii) Model Validation: From the experimental results given in Fig. 7, the parameters derived are $\phi_{C 0}=500, \alpha_{C}=503$, $\beta_{C, \max }=-0.657, \beta_{C, \min }=-0.954, D_{W, \max }=1, D_{W, \min }=$ 0 . Fitting these values into equation $(10 \mathrm{a})$, the luminous flux for the cool-white LED can be predicted for any $D_{C}$ and $D_{W}$. Fig. 9 gives a comparison of the experimentally measured flux and that calculated using equation (10a). The maximum discrepancy between the two is around $13.2 \%$ and it occurs at $D_{C}=0.3$ and $D_{W}=1$. The minimum discrepancy is around $0.14 \%$ and it occurs at $D_{C}=0.7$ and $D_{W}=0$. The averaged discrepancy between the measured and calculated results is around $5.2 \%$. Therefore, it is concluded that the proposed mathematical model is in good agreement with the actual luminous characteristic of the cool-white LED source in the bi-color lamp.

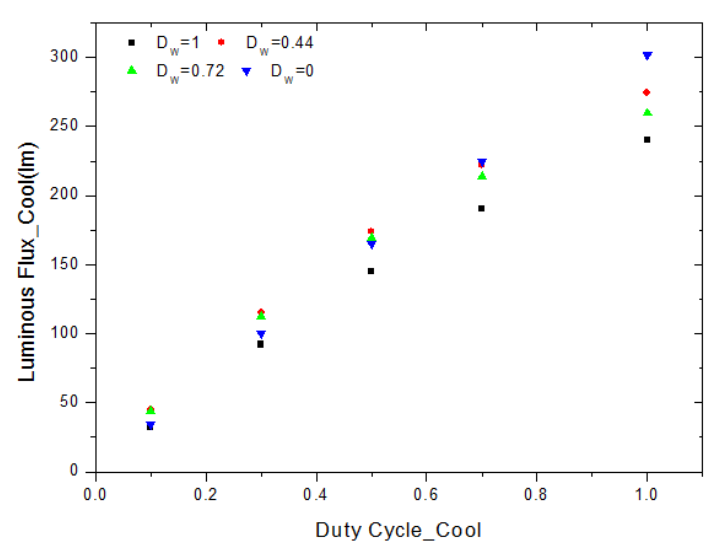

Fig.9. (a) Measured

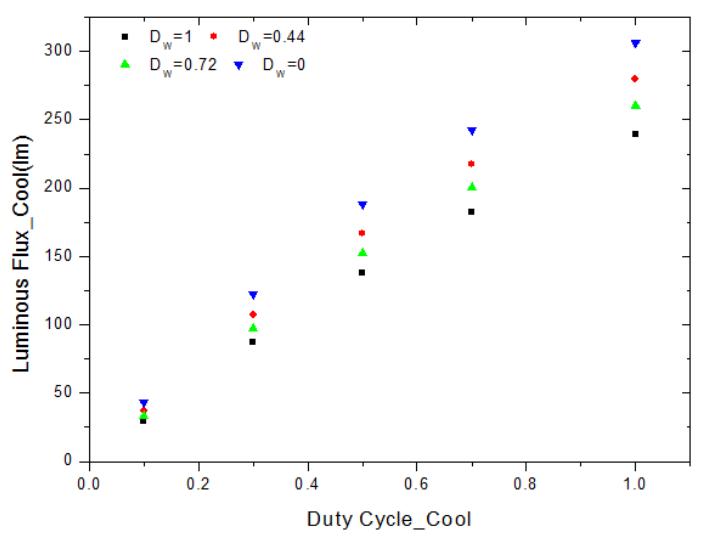

Fig.9. (b) Calculated

Fig. 9. (a) Measured luminous flux and (b) calculated luminous flux of cool-white LED at different $D_{C}$ and $D_{W}$.

From the experimental results given in Fig. 8 , the parameters derived are $\phi_{W 0}=370, \alpha_{W}=376, \beta_{W, \max }=$ $-0.810, \beta_{W, \min }=-0.992, D_{C, \max }=1, D_{C, \min }=0$. Fitting these values into equation (10b), the luminous flux for the warm-white LED can be predicted for any $D_{C}$ and $D_{W}$. Fig. 10 gives a comparison of the experimentally measured flux and that calculated using equation (10b). The maximum discrepancy between the two is around $14.7 \%$ and it occurs at $D_{C}=0$ and $D_{W}=0.1$. The minimum discrepancy is around $0.6 \%$ and it occurs at $D_{C}=1$ and $D_{W}=1$. The averaged discrepancy between the measured and calculated results is around $4.5 \%$. Therefore, it is concluded that the proposed mathematical model is in good agreement with the actual luminous characteristic of the warm-white LED source in the bi-color lamp. 


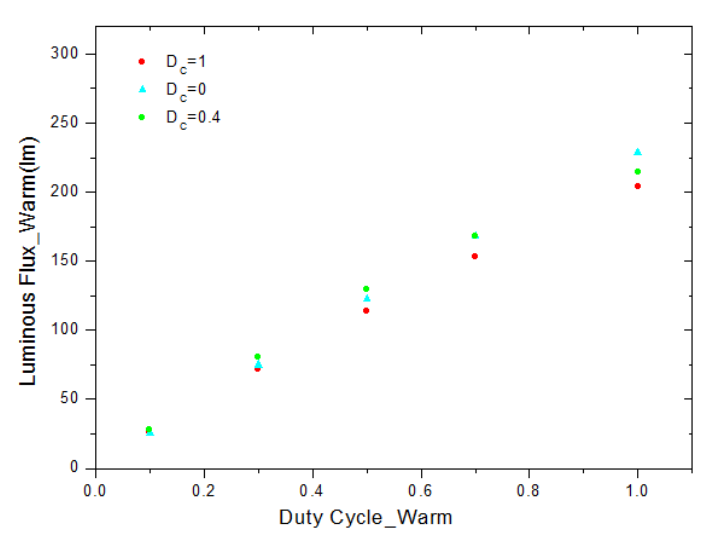

Fig.10. (a) Measured

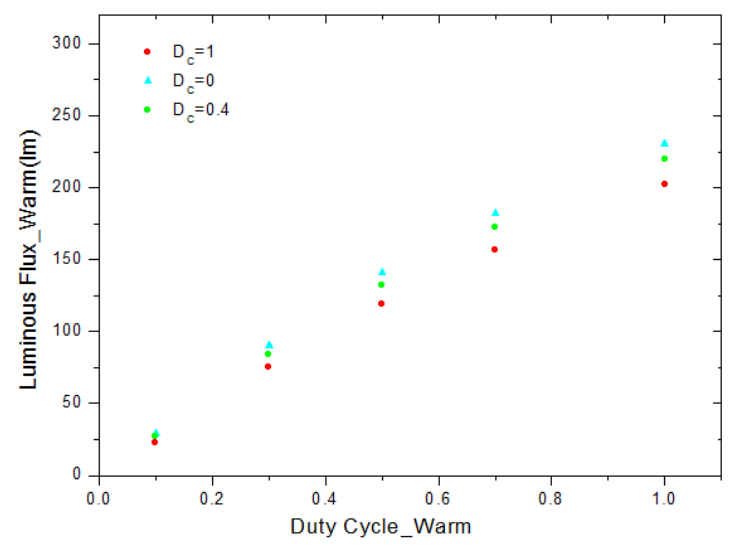

Fig.10. (b) Calculated

Fig. 10. (a) Measured luminous flux and (b) calculated luminous flux of warm-white LED at different $D_{C}$ and $D_{W}$.

\section{Empirical Correlated Color Temperature (CCT) Model of the LEDs in the Bi-Color White LED Lamp}

Similar to the luminous model, the thermal interdependency effect of each LED source on the other will be accounted in the proposed empirical CCT model.

(i) Cool-White LED: With the warm-white LED covered by a black rubber, the maximum and minimum CCT values of the cool-white LED (i.e, $C C T_{C, \max }$ and $C C T_{C, \min }$ ) are measured as a function of the total duty ratio $D_{T}$, where $0 \leq D_{T}=D_{C}+$ $D_{W} \leq 2$. For any value of $D_{T}$, there are two combinations of $D_{C}$ and $D_{W}$ that will each result in a maximum and a minimum CCT value. To obtain the plot of maximum CCT against $D_{T}$, the following equation is considered.

$$
\left(D_{C}, D_{W}\right)_{C C T_{C, \text { max }}}=\left\{\begin{array}{l}
D_{C}=D_{T}, D_{W}=0 ; \text { if } D_{T} \leq 1 \\
D_{C}=1, D_{W}=D_{T}-1 ; \text { if } D_{T}>1
\end{array}\right.
$$

To measure the minimum CCT plot against $D_{T}$, the following equation is considered.

$$
\left(D_{C}, D_{W}\right)_{C C T_{C, \text { min }}}=\left\{\begin{array}{l}
D_{C}=0.1, D_{W}=D_{T}-0.1 ; \quad \text { if } D_{T} \leq 1 \\
D_{C}=D_{T}-1, D_{W}=1 ; \text { if } D_{T}>1
\end{array}\right.
$$

(11b)

With the measured maximum and minimum CCT, the averaged CCT of the cool-white LED at any $D_{T}$ can be calculated using

$$
C C T_{C, \text { ave(measured) }}\left(D_{T}\right)=\frac{C C T_{C, \max \left(D_{T}\right)}+C C T_{C, \min \left(D_{T}\right)}}{2}
$$

Fig. 11 shows a plot of the averaged CCT that is calculated from the measured maximum and minimum CCT using equation (12). Clearly, the graph can be modeled using piecewise linear equations and for this case, the plot is fitted using three straight lines.

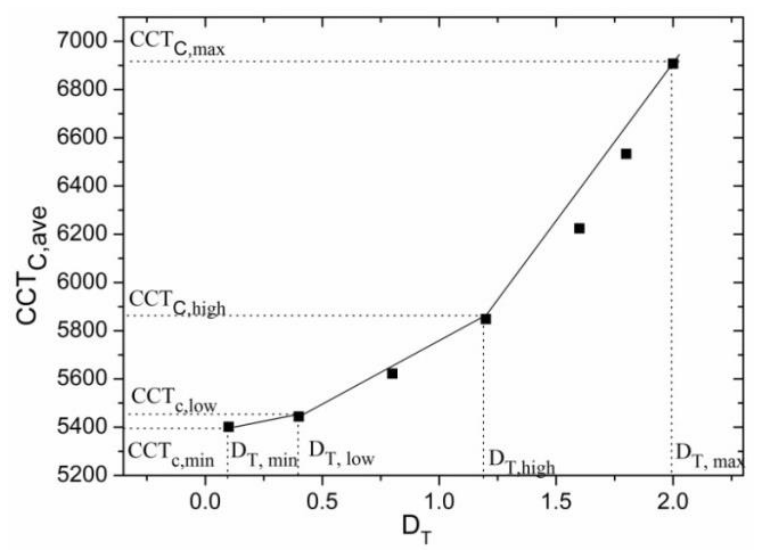

Fig. 11. Plot of the averaged CCT against $D_{T}$ of the cool-white LED fitted by three straight lines.

For the modeling of the averaged CCT using piecewise linear solution as that given in Fig. 11, the following expression can be adopted.

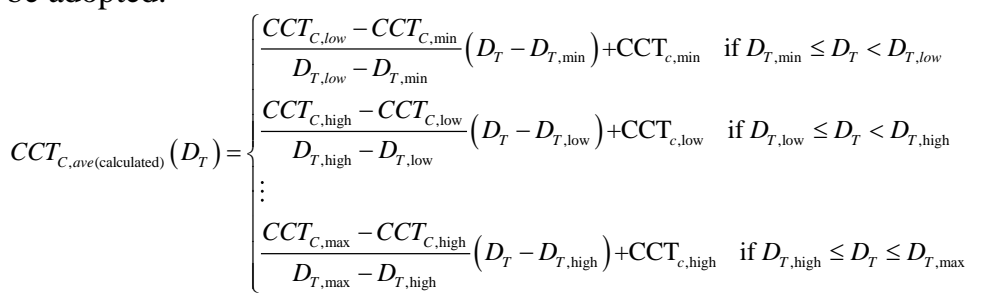

On the other hand, if a more accurate CCT model of the LED is desired, polynomial curve fitting may be used, which will lead to a mathematical expression of this general form

$$
C C T_{C \text {,ave(calculated })}\left(D_{T}\right)=\alpha D_{T}^{2}+\beta D_{T}+\gamma
$$

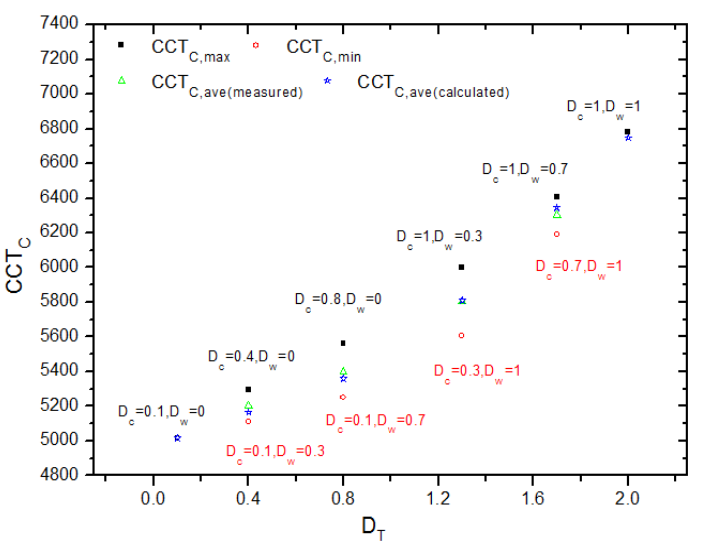


Fig. 12. Measured and calculated CCT of the cool-white LED against $D_{T}$.

Fig. 12 shows the graphs of the measured $C C T_{C, \max }\left(D_{T}\right)$ and $\operatorname{CCT}_{C, \min }\left(D_{T}\right)$ of the cool-white LED, and their averaged values $C C T_{C \text {,ave(measured) }}\left(D_{T}\right)$ (obtained from equation (12)) and $C C T_{C \text {,ave(calculated) }}\left(D_{T}\right)$ (obtained from equation (13)). The maximum and minimum difference between $C_{C} C T_{C, \text { ave (measured) }}\left(D_{T}\right)$ and $C C T_{C, \text { max }}\left(D_{T}\right)$ is about $143 \mathrm{~K}$ (at $D_{T}=1.3$ ) and $36 \mathrm{~K}$ (at $D_{T}=0.4$ ), respectively. Their averaged difference is about $62 \mathrm{~K}$. Since the variations are considerably small (within the perceivable range of $200 \mathrm{~K}$ ), the averaged CCT data, i.e., $C C T_{C \text {,ave(measured) }}\left(D_{T}\right)$ can be used as a simplified representation of the actual CCT characteristics of the cool-white LED. This eases the actual implementation of the control with negligible effect on the quality of its performance. With this as the basis, the parameters can be extracted from the graph of $C C T_{C \text {,ave (measured) }}\left(D_{T}\right)$, as shown in Table 3, and then formulate the correlated mathematical expression representing the CCT characteristic of the cool-white LED. A plot of the CCT value based on equation (13) is given in Fig. 12 and is represented by $C C T_{C \text {,ave(calculated) }}\left(D_{T}\right)$. It is clear that both

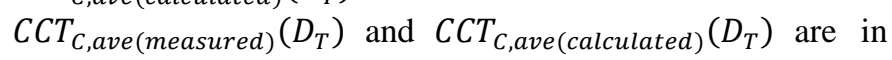
good agreement with negligible discrepancy. Hence, equation (13) can be used for the CCT control of the cool-white LED.

Table. 3. The required parameters in (13) for cool-white LED

\begin{tabular}{|c|c|c|c|c|c|c|c|}
\hline $\begin{array}{c}D_{T,} \\
\text { min }\end{array}$ & $\begin{array}{c}D_{T,} \\
\text { low }\end{array}$ & $\begin{array}{c}D_{T,} \\
\text { high }\end{array}$ & $\begin{array}{c}D_{T,} \\
\max \end{array}$ & $\begin{array}{c}C C T_{C, \min } \\
(\mathrm{K})\end{array}$ & $\begin{array}{c}C C T_{C, \text { low }} \\
(\mathrm{K})\end{array}$ & ${ }^{C C T_{C, \text { high }}}$ & CCT $_{C, \max }$ \\
\hline 0.1 & 0.4 & 1.1 & 2 & 5017 & 5114 & 5543 & 6747 \\
\hline
\end{tabular}

(ii) Warm-White LED: Likewise, with the cool-white LED covered by the black rubber, the maximum and minimum CCT values of the warm-white LED (i.e, $C C T_{W, \max }$ and $C C T_{W, \min }$ ) are measured as a function of the total duty ratio $D_{T}$, where $0 \leq D_{T}=D_{C}+D_{W} \leq 2$. To obtain the plots of maximum and minimum CCT against $D_{T}$, the following equations for setting the duty ratios of the cool-white and warm-white LEDs are considered.

$$
\begin{aligned}
& \left(D_{C}, D_{W}\right)_{C C T_{\mathrm{W}, \text { max }}}=\left\{\begin{array}{l}
D_{W}=D_{T}, D_{C}=0 ; \text { if } D_{T} \leq 1 \\
D_{W}=1, D_{C}=D_{T}-1 ; \text { if } D_{T}>1
\end{array}\right. \\
& \left(D_{C}, D_{W}\right)_{C C T_{\mathrm{W}, \text { min }}}=\left\{\begin{array}{l}
D_{W}=0.1, D_{C}=D_{T}-0.1 ; \text { if } D_{T} \leq 1 \\
D_{W}=D_{T}-1, D_{C}=1 ; \text { if } D_{T}>1
\end{array}\right.
\end{aligned}
$$

Then, the averaged CCT of the warm-white LED can be calculated from

$$
C C T_{W, \text { ave(measured) }}\left(D_{T}\right)=\frac{C C T_{W, \max \left(D_{T}\right)}+C C T_{W, \min \left(D_{T}\right)}}{2}
$$

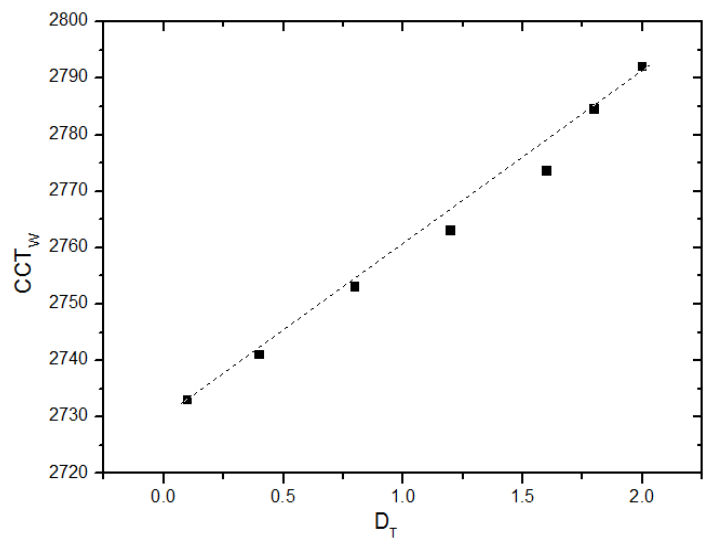

Fig. 13. Plot of the averaged CCT against $D_{T}$ of the warm-white LED fitted by a single straight line.

Fig. 13 shows a graph of the averaged CCT of the warm-white LED that is calculated from the measured maximum and minimum CCT using equation (16). As shown in the figure, the variation of the graph is relatively small and it can be modeled using a straight line with the following expression

$$
C C T_{W, \text { ave }(\text { calculated })}\left(D_{T}\right)=\frac{C C T_{W, \text { max }}-C C T_{W, \text { min }}}{D_{T, \text { max }}-D_{T, \text { min }}}\left(D_{T}-D_{T, \text { min }}\right)+C C T_{W, \text { min }}
$$

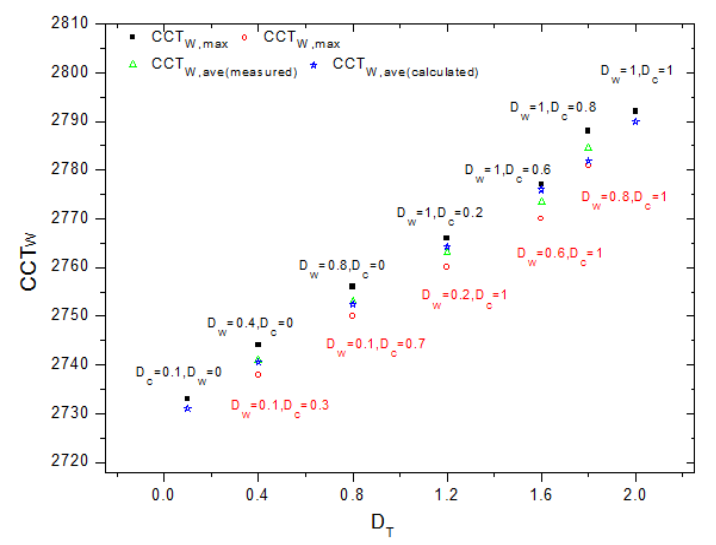

Fig. 14. Measured and calculated CCT of the cool-white LED against $D_{T}$.

Fig. 14 shows the graphs of the measured $C C T_{W, \max }\left(D_{T}\right)$ and $C C T_{W, \min }\left(D_{T}\right)$ of the warm-white LED, and their averaged values $C C T_{W \text {,ave(measured) }}\left(D_{T}\right)$ (obtained from equation (16)) and $C C T_{W \text {,ave (calculated) }}\left(D_{T}\right)$ (obtained from equation (17)). The maximum difference between $C C T_{W \text {,ave(measured) }}\left(D_{T}\right)$ and $C C T_{W, \max }\left(D_{T}\right)$ is about $6 \mathrm{~K}$ (at $D_{T}=0.8$ ). Therefore, the averaged CCT data, i.e., $C C T_{W \text {,ave (measured) }}\left(D_{T}\right)$ can be used as to represent the actual CCT characteristics of the warm-white LED. The required parameters in (17), can be extracted from the graph of $C C T_{W \text {,ave (measured) }}\left(D_{T}\right)$, as shown in Table 4, then formulate the correlated mathematical expression representing the CCT characteristic of the warm-white LED. A plot of the CCT value based on equation (17) is given in Fig. 14 and is represented by $C C T_{W \text {,ave(calculated) }}\left(D_{T}\right)$. It is clear that both $C C T_{W \text {,ave(measured) }}\left(D_{T}\right)$ and $C C T_{W \text {,ave(calculated) }}\left(D_{T}\right)$ are in 
good agreement with negligible discrepancy. Hence, equation (17) can be used for the CCT control of the warm-white LED.

Table. 4. The required parameters in (17) for warm-white LED

\begin{tabular}{|c|c|c|c|}
\hline$D_{T, \min }$ & $D_{T, \max }$ & $C C T_{C, \min }(\mathrm{K})$ & $C C T_{C, \max }(\mathrm{K})$ \\
\hline 0.1 & 2 & 2733 & 2792 \\
\hline
\end{tabular}

\section{Complete Luminous and CCT Model of the Bi-Color White LED Source Lamp}

The total luminous flux $\phi_{M}\left(D_{c}, D_{w}\right)$ of the bi-color LED system is the combined luminous flux of both the warm-white and the cool-white LED and by considering (10a) and (10b), the equation can be expressed as

$$
\begin{aligned}
& \phi_{M}\left(D_{C}, D_{W}\right)=\phi_{c}\left(D_{C}, D_{W}\right)+\phi_{W}\left(D_{C}, D_{W}\right) \\
& =\phi_{C 0}-\alpha_{C} \exp \left\{\left[\frac{\left(\beta_{C, \text { max }}-\beta_{C, \text { min }}\right)}{\left(D_{W, \text { max }}-D_{W, \text { min }}\right)}\left(D_{W}-D_{W, \text { min }}\right)+\beta_{C, \text { min }}\right] D_{C}\right\} \\
& +\phi_{W 0}-\alpha_{W} \exp \left\{\left[\frac{\left(\beta_{W, \text { max }}-\beta_{W, \text { min }}\right)}{\left(D_{C, \text { max }}-D_{C, \text { min }}\right)}\left(D_{C}-D_{C, \text { min }}\right)+\beta_{W, \text { min }}\right] D_{W}\right\}
\end{aligned}
$$

Considering that the CCT of the cool-white LED and the warm-white LED are represented by linear regions as shown in (13) and (17), the mixed $C C T_{M}$ of the bi-color LED system given in (7) can be expressed as

$$
\begin{aligned}
& { }^{C C T_{M}}\left(D_{T}\right)=
\end{aligned}
$$

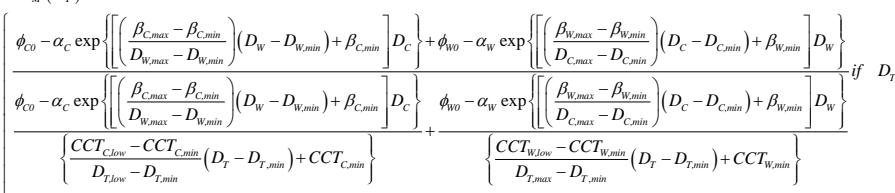

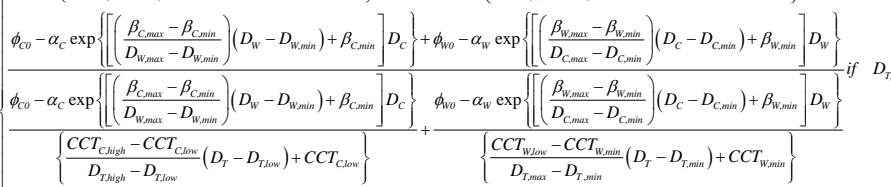

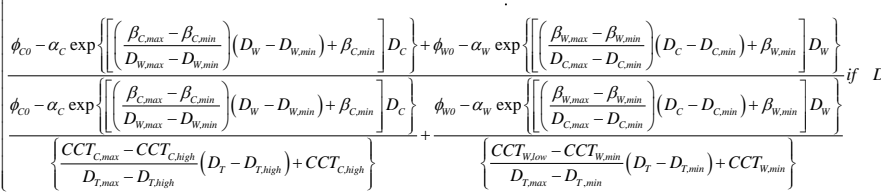

\section{RESULTS AND DISCUSSIONS}

In this section, the results on the application of the nonlinear dimming and CCT control based on the developed empirical CCT and luminous models versus that of the conventional linear approach is reported.

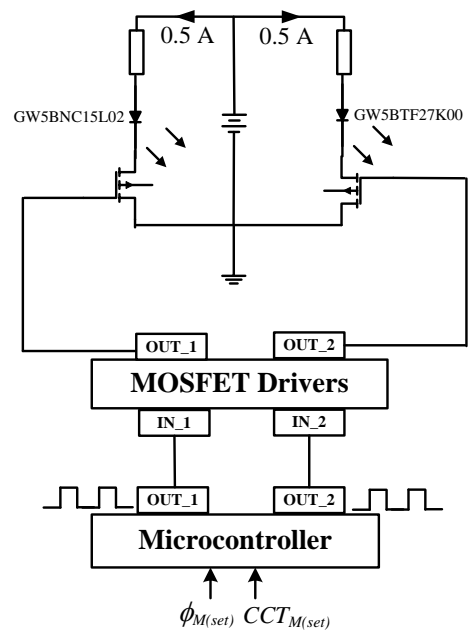

Fig. 15. Basic diagram of the experimental circuit.

Fig. 15 shows the basic diagram of the experimental circuit. With the desired flux $\phi_{M(s e t)}$ and correlated color temperature $C C T_{M(\text { set })}$ being input into the circuit, the microcontroller (STC 11F60XE-35I-PLCC44) which includes a software numerical solver, will generate two individual PWM signals feeding the MOSFET switches through the MOSFET drivers (MC33512), for dimming the cool-white LED (GW5BNC15L02) and the warm-white LED (GW5BTF27K00) to perform the necessary control. The current amplitude of the cool-white and warm-white LEDs are set precisely at $0.5 \mathrm{~A}$ and $0.5 \mathrm{~A}$. Both the LEDs are mounted on the same heatsink which has a thermal resistance of $6.3 \mathrm{~K} / \mathrm{W}$. The combined light of both LED sources $D_{m \text { m }}$ iff sterms of the overall $\phi_{M}$ and $C C T_{M}$ are measured using a PMS-50 Spectro-Photocolorimeter.

\section{B. Numerical Solver}

\section{B. I $_{D}$ For Sharp LED samples}

The purpose of the software numerical solver is to generate the required duty ratio $D_{C}$ and $D_{W}$ for the bi-color lamp to produce the required CCT and flux according to the input values of $\phi_{M(s e t)}$ and $C C T_{M(s e t)}$.

The ${ }^{2}$ substitution of the parameters $\phi_{C 0}=500, \phi_{W 0}=$ $370, \alpha_{C}=503, \alpha_{W}=376, \beta_{C, \max }=-0.657, \beta_{C, \min }=$ $-0.954, D_{W, \max }=1, D_{W, \min }=0, \beta_{W, \max }=-0.81$, $\beta_{W, \text { min }}=-0.992, D_{C, \max }=1, D_{C, \min }=0, D_{T, \min }=$ $0.1, D_{T, \text { low }}=0.4, D_{T, \text { high }}=1.1, D_{T, \max }=2, C C T_{C, \min }=$ $5017, C C T_{C, \text { low }}=5114, C C T_{C, \text { high }}=5543, C C T_{C, \text { max }}=$ $6747, C C T_{W, \min }=2733$, and $C C T_{C, \max }=2792$, which are obtained in the previous sections into equations (18) and (19) give respectively

$$
\begin{aligned}
& \phi_{M}\left(D_{C}, D_{W}\right)=870-503 \exp \left[\left(0.297 D_{W}-0.954\right) D_{C}\right] \\
& -376 \exp \left[\left(0.182 D_{C}-0.992\right) D_{W}\right]
\end{aligned}
$$

\section{A. Experimental Setup}


$C C T_{M}\left(D_{T}\right)=$

$\int \frac{500-503 \exp \left[\left(0.297 D_{W}-0.954\right) D_{C}\right]+370-376 \exp \left[\left(0.182 D_{C}-0.992\right) D_{W}\right]}{\frac{500-503 \exp \left[\left(0.297 D_{W}-0.954\right) D_{C}\right]}{323 D_{T}+4984}+\frac{370-376 \exp \left[\left(0.182 D_{C}-0.992\right) D_{W}\right]}{53.6 D_{T}+2728}}$ if $\quad D_{T, \min } \leq D_{T} \leq D_{T, l, a}$ $\frac{500-503 \exp \left[\left(0.297 D_{W}-0.954\right) D_{C}\right]+370-376 \exp \left[\left(0.182 D_{C}-0.992\right) D_{W}\right]}{500-503 \exp \left[\left(0.297 D_{W}-0.954\right) D_{C}\right] 370-376 \exp \left[\left(0.182 D_{C}-0.992\right) D_{W}\right]}$ if $\quad D_{T, \text { low }} \leq D_{T} \leq D_{T_{-}}$

$\frac{53.6 D_{T}+2728}{613 D_{T}+4868}$

$500-503 \exp \left[\left(0.297 D_{W}-0.954\right) D_{C}\right]+370-376 \exp \left[\left(0.182 D_{C}-0.992\right) D_{W}\right]$ $\frac{500-503 \exp \left[\left(0.297 D_{W}-0.954\right) D_{C}\right]}{1337 D_{T}+4071}+\frac{370-376 \exp \left[\left(0.182 D_{C}-0.992\right) D_{W}\right]}{53.6 D_{T}+2728}$ if $\quad D_{T, h i g h} \leq D_{T} \leq D_{T, \text { m }}$

Then, $D_{C}$ and $D_{W}$ can be solved numerically from (20) and (21). Since there are two equations solving for two unknown variables, the solution is unique. Figs. 16 and 17 depict the complete profile of the luminous flux and CCT values of the bi-color LED system for the full operating range that are calculated using (20) and (21), respectively. For the experiments performed using the nonlinear approach, the microcontroller generates the respective $D_{C}$ and $D_{W}$ by solving equation (20) and (21) based on the input values of $\phi_{M(s e t)}$ and $C C T_{M(s e t)}$. For the experiments performed on the linear approach, the respective $D_{C}$ and $D_{W}$ are generated by solving (20) and (22), which is given as

$$
C C T_{M}=2700 D_{W}+5000 D_{C}
$$

Equation (22) is obtained from equation (1) by setting $C C T_{W}=$ 2700 and $C C T_{C}=5000$.

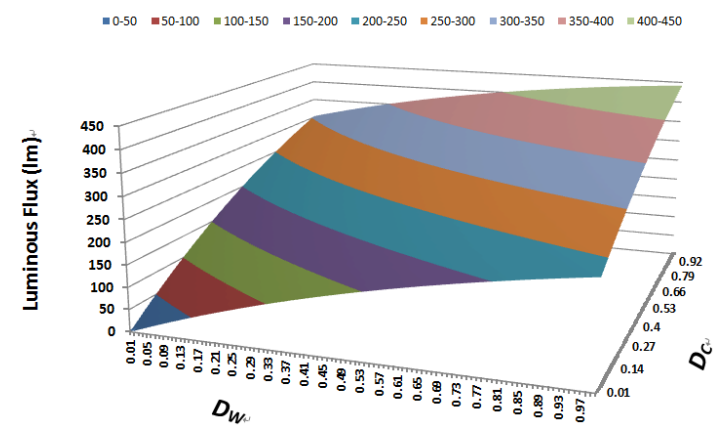

Fig. 16. Profile of the total luminous flux $\phi_{M}$ versus $D_{W}$ and $D_{C}$ calculated using (20).

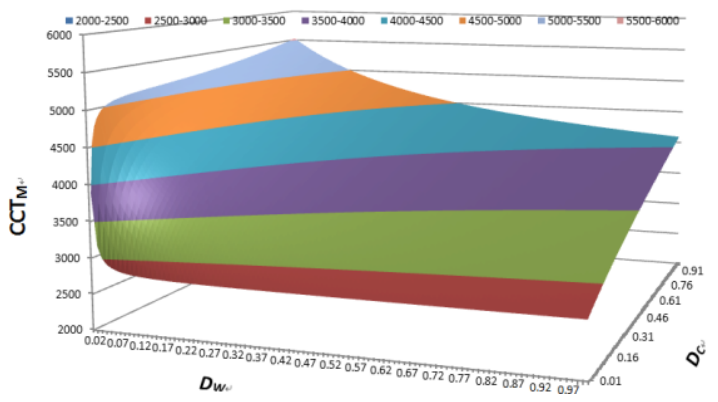

Fig. 17. Profile of $C C T_{M}$ versus $D_{W}$ and $D_{C}$ calculated using (21).

\section{B.2 For CREE LED samples}

In order to confirm proposed method, eight CREE XPE cool-white LEDs (XPEWHT-L1-0000-00C01) and eight
CREE XPE warm-white LEDs (XPEWHT-L1-0000-00BE4) making up the bi-color white LED lamp are mounted on a heatsink with thermal resistance of $2.1 \mathrm{~K} / \mathrm{W}$. According to the datasheets provided, the typical CCT of XPEWHT-L1-0000-00C01 is $7500 \mathrm{~K}$ and that of GW5BTF27K00 is $3000 \mathrm{~K}$. The current amplitude of the cool-white and warm-white LEDs are set precisely at $0.35 \mathrm{~A}$ and $0.35 \mathrm{~A}$.

The substitution of the parameters $\phi_{C 0}=2741 \mathrm{~lm}, \phi_{W 0}=$ $2445 \mathrm{~lm}, \alpha_{C}=2758, \alpha_{W}=2460, \beta_{C, \max }=-0.191$,

$\beta_{C, \min }=-0.232, D_{W, \max }=1, D_{W, \min }=0, \beta_{W, \max }=$

$-0.225, \beta_{W, \min }=-0.235, D_{C, \max }=1, D_{C, \min }=0$,

$D_{T, \text { min }}=0.1, D_{T, \text { low }}=0.4, D_{T, \text { high }}=1.1, D_{T, \max }=$

$2, C C T_{C, \min }=7249 \mathrm{~K}, C C T_{C, \text { low }}=7401 \mathrm{~K}, C C T_{C, \text { high }}=$ $8048 \mathrm{~K}, C C T_{C, \max }=9481 \mathrm{~K}, C C T_{W, \min }=$

$3101 \mathrm{~K}$, and $C C T_{C, \max }=3126 \mathrm{~K}$, which are obtained in the previous sections into equations (18) and (19) give respectively

$$
\begin{aligned}
& \phi_{M}\left(D_{C}, D_{W}\right)=5186-2758 \exp \left[\left(0.041 D_{W}-0.232\right) D_{C}\right] \\
& -2460 \exp \left[\left(0.010 D_{C}-0.235\right) D_{W}\right]
\end{aligned}
$$

$$
\begin{aligned}
& C C T_{M}\left(D_{T}\right)= \\
& \int \frac{2741-2758 \exp \left[\left(0.041 D_{W}-0.232\right) D_{C}\right]+2445-2460 \exp \left[\left(0.010 D_{C}-0.234\right) D_{W}\right]}{\frac{2741-2758 \exp \left[\left(0.041 D_{W}-0.232\right) D_{C}\right]}{508 D_{T}+7194}+\frac{2445-2460 \exp \left[\left(0.010 D_{C}-0.234\right) D_{W}\right]}{12.3 D_{T}+3102}} \text { if } \quad D_{T, \text { min }} \leq D_{T} \leq D_{T, \text { low }} \\
& \frac{2741-2758 \exp \left[\left(0.041 D_{W}-0.232\right) D_{C}\right]+2445-2460 \exp \left[\left(0.010 D_{C}-0.234\right) D_{W}\right]}{2741-2758 \exp \left[\left(0.041 D_{W}-0.232\right) D_{C}\right]}+\frac{2445-2460 \exp \left[\left(0.010 D_{C}-0.234\right) D_{W}\right]}{925 D_{T}+7002} \quad D_{T_{\text {l.ow }}} \leq D_{T} \leq D_{T_{- \text {high }}} \\
& \frac{2741-2758 \exp \left[\left(0.041 D_{W}-0.232\right) D_{C}\right]+2445-2460 \exp \left[\left(0.010 D_{C}-0.234\right) D_{W}\right]}{\frac{2741-2758 \exp \left[\left(0.041 D_{W}-0.232\right) D_{C}\right]}{1591 D_{T}+6249}+\frac{2445-2460 \exp \left[\left(0.010 D_{C}-0.234\right) D_{W}\right]}{12.3 D_{\tau}+3102}} \text { if } \quad D_{T, h \text { high }} \leq D_{T} \leq D_{T, \text { max }}
\end{aligned}
$$

\section{Experimental Results and Discussion}

For the comparison between the proposed nonlinear approach and the linear approach, the following setpoints of luminous flux $\left(\phi_{M(\text { set })}=50 \mathrm{~lm}, 100 \mathrm{~lm}, 150 \mathrm{~lm}, 200 \mathrm{~lm}, 250 \mathrm{~lm}\right.$, and $300 \mathrm{~lm})$ and CCT $\left(C C T_{M(s e t)}=3000 \mathrm{~K}, 3500 \mathrm{~K}, 4000 \mathrm{~K}\right.$, $4500 \mathrm{~K}$, and $5000 \mathrm{~K}$ ) are chosen for the experimental work. Therefore, there are in all 30 possible combinations of target setpoints.

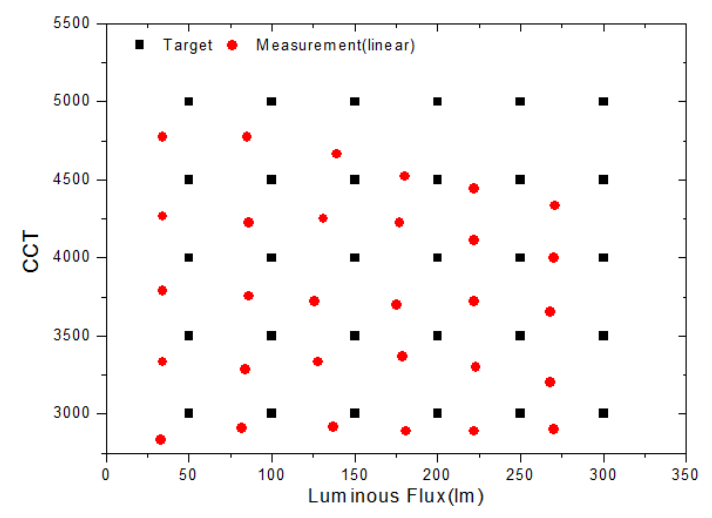

Fig. 18(a) Linear approach 


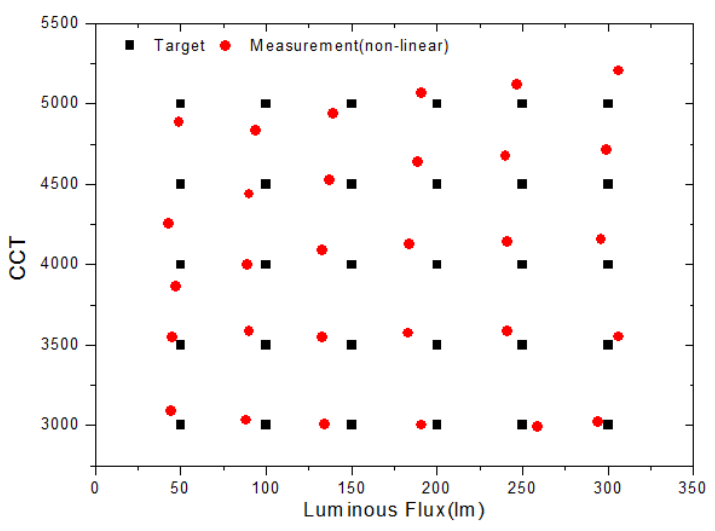

Fig. 18 (b) Nonlinear approach

Fig. 18. Targeted and measured values of the luminous flux and CCT of the bi-color Sharp white LED lamp obtained with (a) the linear approach and (b) the proposed nonlinear approach.

Table 5. Targeted and measured values of the luminous flux and CCT of the bi-color CREE white LED lamp obtained with the linear approach and the proposed nonlinear approach.

\begin{tabular}{|c|c|c|c|c|c|}
\hline \multicolumn{2}{|c|}{ Target Value } & \multicolumn{2}{c|}{$\begin{array}{c}\text { Non-Linear } \\
\text { Approach }\end{array}$} & \multicolumn{2}{c|}{ Linear Approach } \\
\hline $\begin{array}{c}\text { Flux } \\
(\mathrm{lm})\end{array}$ & $\begin{array}{c}\text { CCT } \\
(\mathrm{K})\end{array}$ & Flux (lm) & $\begin{array}{c}\text { CCT } \\
(\mathrm{K})\end{array}$ & $\begin{array}{c}\text { Flux } \\
(\mathrm{lm})\end{array}$ & $\begin{array}{c}\text { CCT } \\
(\mathrm{K})\end{array}$ \\
\hline 100 & 3300 & 95 & 3250 & 88 & 2754 \\
\hline 100 & 5400 & 96 & 5238 & 93 & 4013 \\
\hline 100 & 7500 & 96 & 7394 & 92 & 6493 \\
\hline 300 & 3300 & 296 & 3235 & 258 & 2789 \\
\hline 300 & 5400 & 293 & 5515 & 264 & 4054 \\
\hline 300 & 7500 & 292 & 7654 & 265 & 6687 \\
\hline 550 & 3300 & 562 & 3392 & 455 & 2798 \\
\hline 550 & 5400 & 568 & 5523 & 463 & 4356 \\
\hline 550 & 7500 & 569 & 7729 & 461 & 6843 \\
\hline
\end{tabular}

Table. 6 Nominal CCT Categories (extracted from [41])

\begin{tabular}{|c|c|}
\hline Nominal CCT & $\begin{array}{c}\text { Target CCT and Tolerance } \\
(\mathbf{K})\end{array}$ \\
\hline $2700 \mathrm{~K}$ & $2725 \pm 145$ \\
\hline $3000 \mathrm{~K}$ & $3045 \pm 175$ \\
\hline $3500 \mathrm{~K}$ & $3465 \pm 245$ \\
\hline $4000 \mathrm{~K}$ & $3985 \pm 275$ \\
\hline $4500 \mathrm{~K}$ & $4503 \pm 243$ \\
\hline $5000 \mathrm{~K}$ & $5028 \pm 283$ \\
\hline $5700 \mathrm{~K}$ & $5665 \pm 355$ \\
\hline $6500 \mathrm{~K}$ & $6530 \pm 510$ \\
\hline
\end{tabular}

\section{REFERENCES}

[1] J. Garcia, M. A. Dalla-Costa, J. Cardesin, J. M. Alonso, and M. Rico-Secades, "Dimming of high-brightness LEDs by means of luminous flux thermal estimation," IEEE Trans. Power Electron., vol. 24, no. 4, pp. 1107-1114, Apr. 2009.
Figs. 18(a) and 18(b) depict the experimentally measured values of the luminous flux and CCT of the bi-color Sharp white LED lamp under the 30 target setpoints that are obtained with the linear approach and the proposed nonlinear approach, respectively. As shown in Fig. 18(a), the maximum error between the target setpoints and the experimental results obtained with the linear approach is $22 \mathrm{~lm}(15 \%)$ and $717 \mathrm{~K}$ $(14 \%)$ at the target setpoint $(300 \mathrm{~lm}, 5000 \mathrm{~K})$, as shown in Table 6. Such errors exceed the allowance CCT tolerance according to Table 6 , where the non-perceivable CCT variation at target setpoint $(5000 \mathrm{~K})$ is about $283 \mathrm{~K}$. Their averaged error is about $16 \mathrm{~lm}(10 \%)$ and $202 \mathrm{~K}(5 \%)$. With the nonlinear approach (see Fig. 17(b)), the maximum error between the target setpoint and experimental results is about $17 \mathrm{~lm}(11 \%)$ and $215 \mathrm{~K}(5 \%)$ at the target setpoint $(50 \mathrm{~lm}, 4500 \mathrm{~K})$, as shown in Table 6. Such errors fall within the acceptable CCT tolerance according to Table 6 , where the non-perceivable CCT variation at target setpoint $(4500 \mathrm{~K})$ is about $243 \mathrm{~K}$. Their averaged errors are about $9 \mathrm{~lm}(6 \%)$ and $93 \mathrm{~K}(2 \%)$, respectively. Table 5 shows the measured values of luminous flux and CCT of the bi-color CREE white LED lamp using by nonlinear method has good agreement with target value, compared to linear method. Therefore, it is clearly shown that the nonlinear approach results in a more accurate flux and CCT control of the bi-color variable LED system. While control errors are still present with the nonlinear approach because of model and measurement inaccuracies, these errors in luminance and color is basically non-perceivable and meets the requirement set in the ANSI Standard C78.377 [41] as given in Table 6.

\section{CONCLUSIONS}

A nonlinear approach of controlling the luminous intensity and correlated color temperature (CCT) of bi-color variable CCT white light-emitting diode (LED) lamps is proposed in this paper. The control is built upon nonlinear empirical flux and CCT models of the LEDs that are developed from the experimental results obtained in this work. It is demonstrated that these nonlinear models are easily simplified into practical solutions that are appropriate for implementations. Experimental results verified that the proposed approach is considerably more accurate than existing linear approaches in obtaining the desired brightness and CCT control in bi-color LED lamps. This approach is applicable to lamps with LEDs of multiple color temperatures.

\section{ACKNOWLEDGMENT}

This project is supported by the Hong Kong Research Grant Council under the Theme-based Research Scheme (T22-715/12-N).

[2] M. Dyble, N. Narendran, A. Bierman, and T. Klein, "Impact of dimming white LEDs: Chromaticity shifts due to different dimming methods," Proc. SPIE, vol. 5941 pp. 291-299, 2005.

[3] Y. Hu and M. M. Jovanovic, "LED driver with self-adaptive drive voltage (efficiency and dimming)," 
IEEE Trans. Power Electron., vol. 23, no. 6, pp. 31163125, Mar. 2008.

[4] W. Yu, J. S. Lai, H. Ma, C. Zheng, "High-efficiency DCDC converter with twin bus for dimmable LED lighting," IEEE Trans. Power Electron., vol. 26, no. 8, pp. 20952100, Aug. 2011.

[5] Y. T. Huang, Y. T. Chen, Y. H. Liu, H. C. Hsiao, and W. T. Tsai, "Compact-size and high-conversion-efficiency regulator for alternating-current-operated light-emitting diodes," IEEE Trans. Indust. Electron., vol. 58, no. 9, pp. 4130-4135, Sep. 2011.

[6] Y. C. Li and C. L. Chen, "A novel single-stage high-power-factor AC-to-DC LED driving circuit with leakage inductance energy recycling," IEEE Trans. Indust. Electron., vol. 59, no. 2, pp. 793-802, Feb. 2012.

[7] M. Arias, D. G. Lamar, F. F. Linera, D. Balocco, A. A. Diallo, and J. Sebastian, "Design of a soft-switching asymmetrical half-bridge converter as second stage of an LED driver for street lighting application," IEEE Trans. Power Electron., vol. 27, no. 3, pp. 1608-1621, Mar. 2012.

[8] C. S. Moo, Y. J. Chen, and W. C. Yang, "An efficient driver for dimmable LED lighting," IEEE Trans. Power Electron., vol. 27, no. 11, pp. 4613-4618, Nov. 2012.

[9] S. Buso, G. Spiazzi, M. Meneghini, and G. Meneghesso, "Performance degradation of high-brightness light emitting diodes under DC and pulsed bias," IEEE Trans. Device Mater. Rel., vol. 8, no. 2, pp. 312-322, Jun. 2008.

[10] H. J. Chiu, Y. K. Lo, J. T. Chen, S. J. Cheng, and C. Y. Lin, S. C. Mou, "A high-efficiency dimmable LED driver for low-power lighting applications (efficiency)," IEEE Trans. Indust. Electron., vol. 57, no. 2, pp. 735-743, Feb. 2010.

[11] J. Garcia, A. J. Calleja, E. Lopez rominas, D. Gacio Vaquero, and L. Campa, "Interleaved buck converter for fast PWM dimming of high-brightness LEDs," IEEE Trans. Power Electron., vol. 26, no. 9, pp. 2627-2636, Sep. 2011.

[12]D. Gacio, J. M. Alonso, J. Garcia, L. Campa, M. J. Crespo, and M. Rico-Secades, "PWM series dimming for slow-dynamics HPF LED drivers: the high-frequency approach," IEEE Trans. Indust. Electron., vol. 59, no. 4, pp. 1717-1727, Apr. 2012.

[13] J. Zhang, H. Zeng, T. Jiang, "A primary-side control scheme for high-power-factor LED driver with TRIAC dimming capability," IEEE Trans. Power Electron., vol. 27, no. 11, pp. 4619-4629, Nov. 2012.

[14]R. Zhang and H. S. Chung, "A TRIAC-dimmable LED lamp driver with wide dimming range," IEEE Trans. Power Electron., vol. 29, no. 3, pp. 1434-1446, Mar. 2014.

[15] L. Gu, X. Ruan, M. Xu, and K. Yao, "Means of eliminating electrolytic capacitor in AC/DC power supplies for LED lightings," IEEE Trans. Power Electron., vol. 24, no. 5, pp. 1399-1408, May. 2009.

[16]B. Wang, X. Ruan, K. Yao, and M. Xu, "A method of reducing the peak-to-average ratio of LED current for electrolytic capacitor-less AC-DC drivers," IEEE Trans. Power Electron., vol. 25, no. 3, pp. 592-601, Mar. 2010.

[17] S. Y. R. Hui, S. N. Li, X. Tao, W. Chen, and W. M. Ng, "A novel passive offline LED driver with long lifetime," IEEE Trans. Power Electron., vol. 25, no. 10, pp. 2665-2672, Oct. 2010.

[18]X. Ruan, B. Wang, K. Yao, and S. Wang, "Optimum injected current harmonics to minimize peak-to-average ratio of LED current for electrolytic capacitor-less AC-DC drivers," IEEE Trans. Power Electron., vol. 26, no. 7, pp. 1820-1825, Jul. 2011.

[19] Q. Hu and R. Zane, "Minimizing required energy storage in off-line LED drivers based on series-input converter modules," IEEE Trans. Power Electron., vol. 26, no. 10, pp. 2887-2895, Oct. 2011.

[20] J. M. Alonso, J. Viña, D. G. Vaquero, G. Martinez, and R. Osorio, "Analysis and design of the integrated double buck-boost converter as a high-power-factor driver for power-LED lamps," IEEE Trans. Indust. Electron., vol. 59, no. 4, pp. 1689-1697, Apr. 2012.

[21]F. Zhang, J. Ni, and Y. Yu, "High power factor AC-DC LED driver with film capacitors," IEEE Trans. Power Electron., vol. 28, no. 10, pp. 4831-4840, Oct. 2013.

[22] S. C. Tan, "General $n$-level driving approach for improving electrical-to-optical energy-conversion efficiency of fast-response saturable lighting devices," IEEE Trans. Industral. Electron., vol. 57, no. 4, pp. 1342-1353, Apr. 2010.

[23]A. Wilkins, J. Veitch, and B. Lehman, "LED lighting flicker and potential health concerns: IEEE standard PAR1789 update," in Proc. IEEE ECCE, Sep. 2010, pp. 171-178.

[24] B. Lehman, A. Wilkins, S. Berman, M. Poplawski, and N. J. Miller, "Proposing measures of flicker in the low frequencies for lighting applications," in Proc. IEEE ECCE, , Sep. 2011, pp. 2865-2872.

[25] W. Chen and S. Y. R. Hui, "Elimination of an electrolytic capacitor in AC/DC light-emitting diode (LED) driver with high input power factor and constant output current," IEEE Trans. Power Electron., vol. 27, no. 3, pp. 1598-1607, Mar. 2012.

[26] S. Wang, X. Ruan, K. Yao, S. -C. Tan, Y. Yang, and Z. Ye, "A flicker-free electrolytic capacitor-less AC-DC LED driver," IEEE Trans. Power Electron., vol. 27, no. 11, pp. 4540-4549, Mar. 2012.

[27]H. Ma, J. -S. Lai, Q. Feng, W. Yu, C. Zheng, and Z. Zhao, "A novel valley-fill SEPIC-derived power supply without electrolytic capacitor for LED lighting application," IEEE Trans. Power Electron., vol. 27, no. 6, pp. 3057-3071, Jun. 2012.

[28] M. Arias, D. M. Fernandez, D. G. Lamar, D. Balocco, A. A. Diallo, and J. Sebastian, "High-efficiency asymmetrical 
half-bridge converter without electrolytic capacitor for low-output-voltage AC-DC LED drivers," IEEE Trans. Power Electron., vol. 28, no. 5, pp. 2539-2550, May 2013.

[29]R. Paschotta, Encyclopedia of Laser Physics and Technology. Wiley-VCH, pp. 219, 2008.

[30] P. R. Mills, S. C. Tomkins, and L. J. M. Schlangen, "The effect of high correlated colour temperature office lighting on employee wellbeing and work performance," Journal of Circadian Rhythms., no. 5, vol. 2, Jan. 2007.

[31] T. Nimz, F. Hailer, and K. Jensen, Sensors and Feedback Control of Multi-Color LED Systems. LED Professional, pp. 2-5, 2012.

[32] L. Li, "Why color temperature control is vital to the LED industry," in LED, Recent Publications \& Product News, Mar. $2013.2 \quad$ (Online: http://www.cleantechpatentedge.com/2013/03/why-color-t emperature-control-is-vital-to-the-led-industry/)

[33] C. H. Miao, “Color temperature adjustable lamp," United State Patent: US8159125B2.

[34] K. Jonsson, "Light with changeable color temperature," United State Patent: US20120146505A1.

[35] O. Noboru. Colorimetry: fundamentals and applications, John Wiley \& Sons, 2006.

[36] Y. Gu, N. Narendran, T. Dong, and H. Wu, "Spectral and luminous efficacy change of high-power LEDs under different dimming methods," Proceedings of SPIE., 2006.

[37]H. T. Chen, S. C. Tan, and S. Y. R. Hui, "Correlated color temperature control system and method," PCT Application: PCT/CN2014/078552, 27 May 2014.

[38]H. T. Chen and S. Y. R. Hui, "Dynamic prediction of correlated color temperature and color rendering index of phosphor-coated white light-emitting diodes", IEEE Trans. Indust. Electron., vol. 61, no.2, pp. 784-797, Feb, 2014.

[39] H. T. Chen, S. C. Tan, and S. Y. R. Hui, "Color variation reduction of GaN-based white light-emitting diodes via peak-wavelength stabilization", IEEE Trans. Power Electron., vol.29, no.7, pp. 3709-3719, Jul, 2014.

[40] H. K. Fu, C. W. Lin, T. T. Chen, C. L. Chen, P. T. Chou, and C. J. Sun, "Investigation of dynamic color deviation mechanisms of high power light-emitting diode," Microelectronics Reliability, vol. 52, pp. 866-871, 2012.

[41]American National Standard, Specifications for Chromaticity of Solid State Lighting Products, ANSI C78.377 (American National Standards Institute, 2008).

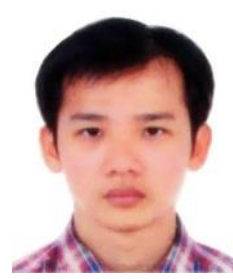

Huanting Chen (M'13) received the Ph.D degree in radio physics from Xiamen Unviersity, Xiamen, China, in 2010. He was a Joint Ph.D student at the Light \& Lighting Laboratory, Catholic University College Gent, Belgium, from November 2009 to May 2010. He was a Senior Research Associate in the Departement of Electronic Engineering, City University of Hong Kong, Hong Kong, in 2011. He is currently Postdoctoral Fellow in Department of Electrical and Electronic Engineering, The University of Hong Kong, Hong Kong. His research intersts include solid-state lighting theory and techonolgy.

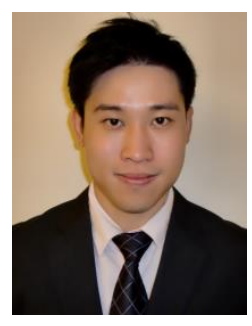

Siew-Chong Tan (S'00-M'06-SM'11) received the B.Eng. (Hons.) and M.Eng. degrees in electrical and computer engineering from the National University of Singapore, Singapore, in 2000 and 2002, respectively, and the Ph.D. degree in electronic and information engineering from the Hong Kong Polytechnic University, Hong Kong, in 2005.

From October 2005 to May 2012, he worked as Research Associate, Postdoctoral Fellow, Lecturer, and Assistant Professor in Department of Electronic and Information Engineering, Hong Kong Polytechnic University, Hong Kong. From January to October 2011, he was Senior Scientist in Agency for Science, Technology and Research (A*Star), Singapore. He is currently an Associate Professor in Department of Electrical and Electronic Engineering, The University of Hong Kong, Hong Kong. Dr. Tan was a Visiting Scholar at Grainger Center for Electric Machinery and Electromechanics, University of Illinois at Urbana-Champaign, Champaign, from September to October 2009, and an Invited Academic Visitor of Huazhong University of Science and Technology, Wuhan, China, in December 2011. His research interests are focused in the areas of power electronics and control, LED lightings, smart grids, and clean energy technologies.

Dr. Tan serves extensively as a reviewer for various IEEE/IET transactions and journals on power, electronics, circuits, and control engineering. He is an Associate Editor of the IEEE Transactions on Power Electronics. He is a coauthor of the book Sliding Mode Control of Switching Power Converters: Techniques and Implementation (Boca Raton: CRC, 2011).

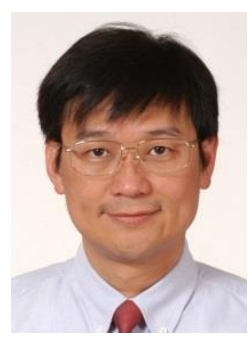

S. Y. (Ron) Hui (M'87-SM'94-F'03) received his BSc (Eng) Hons at the University of Birmingham in 1984 and a D.I.C. and PhD at Imperial College London in 1987. He has previously held academic positions at the University of Nottingham (1987-90), University of Technology Sydney (1991-92), University of Sydney (92-96), City University of Hong Kong (1996-2011). Presently, he holds the Chair Professorships at the University of Hong Kong and Imperial College London.

He has published over 200 technical papers, including more than 170 refereed journal publications and book chapters. Over 55 of his patents have been adopted by industry. He has been an Associate Editor of the IEEE Transactions on Power Electronics since 1997 and an Associate Editor of the IEEE Transactions on Industrial Electronics since 2007. He has been appointed twice as an IEEE Distinguished Lecturer by the IEEE Power Electronics Society in 2004 and 2006. He served an AdCom member of the IEEE Power Electronics Society and was the Chairman of its Constitution and Bylaws Committee from 2002-2010. He received the Excellent Teaching Award in 1998 and the Earth Champion Award in 2008. He won an IEEE Best Paper Award from the IEEE IAS Committee on Production and Applications of Light in 2002, and two IEEE Power Electronics Transactions Prize Paper Awards for his publications on Wireless Charging Platform Technology in 2009 and on LED system theory in 2010. His inventions on wireless charging platform technology underpin key dimensions of Qi, the world's first wireless power standard, with freedom of positioning and localized charging features for wireless charging of consumer electronics. In Nov. 2010, he received the IEEE Rudolf Chope R\&D Award from the IEEE Industrial Electronics Society, the IET Achievement Medal (The Crompton Medal) and was elected to the Fellowship of the Australian Academy of Technological Sciences \& Engineering and the recipient of the 2015 IEEE William E. Newell Award. 